\title{
When an Extension of Nagata Rings Has Only Finitely Many Intermediate Rings, Each of Those Is a Nagata Ring
}

\author{
David E. Dobbs, ${ }^{1}$ Gabriel Picavet, ${ }^{2}$ and Martine Picavet-L'Hermitte ${ }^{2}$ \\ ${ }^{1}$ Department of Mathematics, University of Tennessee, Knoxville, TN 37996-1320, USA \\ ${ }^{2}$ Laboratoire de Mathématiques, Université Blaise Pascal, UMR6620, CNRS, Les Cézeaux, 24 avenue des Landais, \\ BP 80026, 63177 Aubière Cedex, France
}

Correspondence should be addressed to David E. Dobbs; dobbs@math.utk.edu

Received 30 March 2014; Accepted 14 June 2014; Published 2 September 2014

Academic Editor: Kaiming Zhao

Copyright (C) 2014 David E. Dobbs et al. This is an open access article distributed under the Creative Commons Attribution License, which permits unrestricted use, distribution, and reproduction in any medium, provided the original work is properly cited.

Let $R \subset S$ be an extension of commutative rings, with $X$ an indeterminate, such that the extension $R(X) \subset S(X)$ of Nagata rings has FIP (i.e., $S(X)$ has only finitely many $R(X)$-subalgebras). Then, the number of $R(X)$-subalgebras of $S(X)$ equals the number of $R$-subalgebras of $S$. In fact, the function from the set of $R$-subalgebras of $S$ to the set of $R(X)$-subalgebras of $S(X)$ given by $T \mapsto T(X)$ is an order-isomorphism.

\section{Introduction and Notation}

All rings considered below are commutative and unital; all inclusions of rings and all ring homomorphisms are unital. As usual, if $R$ is a ring, then $\operatorname{Spec}(R)$ and $\operatorname{Max}(R)$ denote the sets of prime ideals of $R$ and of maximal ideals of $R$, respectively; if $P \in \operatorname{Spec}(R)$, then $\kappa(P):=R_{P} / P R_{P}$; if $R \subseteq S$ is an (ring) extension, then $(R: S):=\{s \in S \mid s S \subseteq R\}$, the conductor of $R \subseteq S$; and if $f: R \rightarrow S$ is a ring homomorphism, then ${ }^{a} f$ denotes the canonical map $\operatorname{Spec}(S) \rightarrow \operatorname{Spec}(R)$, $Q \mapsto f^{-1}(Q)$. As in [1], the support of an $R$-module $E$ is the set $\operatorname{Supp}(E):=\left\{P \in \operatorname{Spec}(R) \mid E_{P}\left(:=E_{R \backslash P}\right) \neq 0\right\}$ and $\operatorname{MSupp}(E):=\operatorname{Supp}(E) \cap \operatorname{Max}(R)$. Also as usual, if $I$ is an ideal of a ring $R$, then $\mathrm{V}(I):=\mathrm{V}_{R}(I):=\{P \in \operatorname{Spec}(R) \mid I \subseteq P\}$; and $|Y|$ denotes the cardinality of a set $Y$.

Let $R \subseteq S$ be an (ring) extension. The set of all (unital) $R$-subalgebras of $S$ is denoted by $[R, S]$. Following [2], the extension $R \subseteq S$ is said to have (or to satisfy) FIP (for the "finitely many intermediate algebras property") if $[R, S]$ is finite. As usual, by a chain of $R$-subalgebras of $S$, we mean a set of elements of $[R, S]$ that are pairwise comparable with respect to inclusion. Recall that the extension $R \subseteq S$ has (or satisfies) FCP (for the "finite chain property") if each chain of $R$-subalgebras of $S$ is finite. It is clear that FIP implies FCP. We will freely use the characterizations of the FCP extensions and of the FIP extensions that were given in [1].
Minimal (ring) extensions, as introduced by Ferrand and Olivier [3], are our main tool for studying the FIP and FCP properties. Recall that an extension $R \subset S$ is called minimal if $[R, S]=\{R, S\}$. (Note that since $C$ denotes proper inclusion, $R \neq S$ whenever $R \subseteq S$ is a minimal extension.) The key connection between the above ideas is that if $R \subseteq S$ has FCP, then each maximal (necessarily finite) chain of $R$-subalgebras of $S$ can be written as $R=R_{0} \subseteq R_{1} \subseteq \cdots \subseteq R_{n-1} \subseteq R_{n}=S$, with length $n$, where $0 \leq n<\infty$, and results from juxtaposing $n$ minimal extensions $R_{i} \subset R_{i+1}, 0 \leq i \leq n-1$. For any extension $R \subseteq S$, the length of $[R, S]$, denoted by $\ell[R, S]$, is the supremum of the lengths of chains of $R$-subalgebras of $S$.

The following notions are also deeply involved in our study.

Definition 1 (see [1, Definition 4.4]). Let $f: R \hookrightarrow S$ be an integral extension. Then $f$ is called infra-integral if for each $Q \in \operatorname{Spec}(S)$, the residual extension $\kappa\left(f^{-1}(Q)\right) \hookrightarrow \kappa(Q)$ is an isomorphism. Moreover, $f$ is called subintegral if $f$ is infraintegral and ${ }^{a} f$ is a bijection.

Consider an extension $R \subseteq S$. Then $R \subseteq S$ is called $t$-closed if the relations $b \in S, r \in R, b^{2}-r b \in R$, and $b^{3}-r b^{2} \in R$ imply $b \in R$. Also, $R \subseteq S$ is called seminormal if the relations $b \in S$, $b^{2} \in R$, and $b^{3} \in R$ imply $b \in R$. If $R \subset S$ is seminormal, $(R: S)$ is a radical ideal of $S$. The $t$-closure of $R$ in $S$, denoted by ${ }_{S}^{t} R$, is 
the smallest $R$-subalgebra $B$ of $S$ such that $B \subseteq S$ is $t$-closed, as well as the greatest $R$-subalgebra $C$ of $S$ such that $R \subseteq C$ is infra-integral. The seminormalization of $R$ in $S$, denoted by ${ }_{S}^{+} R$, is the smallest $R$-subalgebra $B$ of $S$ such that $B \subseteq S$ is seminormal, as well as the greatest $R$-subalgebra $C$ of $S$ such that $R \subseteq C$ is subintegral. The chain $R \subseteq{ }_{S}^{+} R \subseteq{ }_{S}^{t} R \subseteq \bar{R} \subseteq S$ is called the canonical decomposition of $R \subseteq S$, where $\bar{R}$ denotes the integral closure of $R$ in $S$.

Let $R$ be a ring and $R[X]$ the polynomial ring in the indeterminate $X$ over $R$. (Throughout, we use $X$ to denote an element that is indeterminate over all relevant coefficient rings.) Also, let $C(p)$ denote the content of any polynomial $p(X) \in R[X]$. Then $\Sigma_{R}:=\{p(X) \in R[X] \mid C(p)=R\}$ is a saturated multiplicatively closed subset of $R[X]$, each of whose elements is a non-zero-divisor of $R[X]$. The Nagata ring of $R$ is defined to be $R(X):=R[X]_{\Sigma_{R}}$. Let $R \subseteq S$ be an extension. It was shown in [4, Theorem 3.9] that $R(X) \subseteq S(X)$ has FCP if and only if $R \subseteq S$ has FCP. The analogous assertion does not hold in general for the FIP property. One implication does hold in general, as it was shown in [4, Proposition 3.2] that if $R(X) \subseteq S(X)$ has FIP, then $R \subseteq S$ must also have FIP. We next recall some partial converses to the preceding assertion.

Let $R \subset S$ be an FIP extension. By [4, Theorem 3.21], $R(X) \subset S(X)$ has FIP if and only if $R(X) \subseteq{ }_{\bar{R}}^{+}(X) R(X)$ has FIP. This last condition holds when $|R / M|=\infty$ for each $M \epsilon$ $\operatorname{MSupp}\left({ }_{R}^{+} R / R\right)$, by [4, Corollary 3.25]. As $|R(X) / M R(X)|=$ $|(R / M)(X)|=\infty$ for each $M \in \operatorname{Max}(R)$, it follows that FIP satisfies the following analogue of a result on FCP [4, Corollary 3.10]: $R(X) \subseteq S(X)$ has FIP if and only if $R\left(X_{1}, \ldots, X_{n}\right) \subseteq S\left(X_{1}, \ldots, X_{n}\right)$ has FIP for some (resp., each) positive integer $n$.

One can say more along these lines. By using results from $[1,4]$, we will obtain, in Theorem 2 , a new characterization of when FIP holds for $R(X) \subset S(X)$. Let us say that a ring extension $R \subset S$, with seminormalization $T:={ }^{+} R$, satisfies the property $(*)$ if for each $M \in \operatorname{MSupp}(T / R)$ such that $|R / M|<\infty$, one has that $\left[\left(R_{M}\right)_{2}, T_{M}\right]$ is linearly ordered and $L_{R_{M}}\left(\left(M T_{M}\right) /\left(M R_{M}\right)\right)=n_{M}-1$, where $n_{M}$ denotes the nilpotency index of $R_{M} /\left(R_{M}: T_{M}\right)$ and $\left(R_{M}\right)_{2}:=R_{M}+$ $M^{2} T_{M}$.

Theorem 2. Let $R \subset S$ be a ring extension. Then $R(X) \subset S(X)$ has FIP if and only if $R \subset S$ has FIP and satisfies (*).

Proof. Combine [1, Corollary 3.2 and Proposition 3.7(a)] with [4, Corollary 3.25 and Theorem 3.30].

In regard to an extension $R \subseteq S$, our main concern here is, as it was in $[4$, Section 4$]$, the function $\varphi:[R, S] \rightarrow$ $[R(X), S(X)]$, defined by $T \mapsto T(X)$. Our goal, which will be accomplished in Theorem 32, is to show that if $R(X) \subseteq$ $S(X)$ has FIP (in which case, $R \subseteq S$ must also have FIP), then $\varphi$ is an order-isomorphism. Since $\varphi$ is known to be an order-preserving and order-reflecting injection [4, Lemma 3.1(d)] in general, it remains only to show that $\varphi$ is surjective (assuming that $R(X) \subseteq S(X)$ has FIP). Evidence for Theorem 32 was provided in [4, Propositions 4.4, 4.14, 4.17], where it was shown that if $R \subseteq S$ has FIP, then $\varphi$ is an order-isomorphism in the following three cases: $R \subseteq S$ is an integrally closed extension; $R \subseteq S$ is a subintegral extension such that $R(X) \subset S(X)$ has FIP; $R \subseteq S$ is a seminormal infraintegral extension. Thus, in view of the steps in the canonical decomposition of an extension $R \subseteq S$, it is clear that [4, Section 4] failed to make much headway for the case of an integral $t$-closed extension. In fact, as summarized next, our path to Theorem 32 will rely on a deeper study of precisely such extensions.

It is easy to see that any extension of fields is $t$-closed. We begin Section 2 by showing in Propositions 9 and 11 that if $K \subseteq L$ is an FIP field extension (hence, an integral $t$-closed extension), then $\varphi:[K, L] \rightarrow[K(X), L(X)]$ is an orderisomorphism. This fact is used in the proof of Theorem 12, which obtains an affirmative answer to our main question in case of an arbitrary integral $t$-closed extension $R \subseteq S$. The arguments in Section 3 proceed with an eye on the steps in the canonical decomposition of an extension $R \subseteq S$ and the four types of minimal extensions (which are reviewed later in this Introduction). In case $R(X) \subset S(X)$ has FIP, we establish the nature of a minimal subextension of $R(S) \subset S(X)$, first for the case of a quasi-local base ring $R$ in Proposition 30 and then in general in Proposition 31. Our main result is then obtained in Theorem 32 by an inductive argument.

It is convenient to close the Introduction by stating some results that summarize the fundamental facts about minimal extensions, FCP extensions, and FIP extensions that we will use below.

Theorem 3 (see [5], [6, Theorem 4.1], [3, Théorème 2.2 and Lemme 3.2], [7, Proposition 3.2], [4, Theorem 1.1]). Let $A \subset B$ be a minimal extension with associated inclusion map $f: A \rightarrow$ B. Then,

(a) there is some $M \in \operatorname{Max}(A)$, called the crucial (maximal) ideal of $A \subset B$, such that $A_{P}=B_{P}$ for each $P \in$ $\operatorname{Spec}(A) \backslash\{M\}$. We denote this ideal $M$ by $\mathscr{C}(A, B)$;

(b) with $f$ and $M$ as above, the following three conditions are equivalent:

(1) some prime ideal of $B$ lies over $M$;

(2) $M B=M$;

(3) $f$ is (module-) finite;

(c) the (equivalent) conditions in (b) do not hold if and only if $f$ is a flat epimorphism (in the sense of [8]); and, in that case, $(A: B)$ is a common prime ideal of $A$ and $B$ that is contained in $M$;

(d) there is a bijection $\operatorname{Spec}(B) \backslash V(M B) \rightarrow \operatorname{Spec}(A) \backslash$ $\{M\}$, with $V(M B)=\emptyset$ when $f$ is a flat epimorphism. Moreover, if $Q \in \operatorname{Max}(B)$, then either $Q=(A: B)$ or $Q \cap A \in \operatorname{Max}(A)$.

There are three types of integral minimal extensions, as given in Theorem 4 . Thus, by also counting the flat epimorphisms discussed in Theorem 3(c), there are four types of minimal extensions. 
Theorem 4 (see [7, Theorem 3.3]). Let $R \subset T$ be an extension and let $M:=(R: T)$. Then, $R \subset T$ is minimal and finite (i.e., an integral minimal extension) if and only if $M \in \operatorname{Max}(R)$ and (exactly) one of the following three conditions holds:

(a) inert case: $M \in \operatorname{Max}(T)$ and $R / M \rightarrow T / M$ is a minimal field extension.

(b) decomposed case: there exist $M_{1}, M_{2} \in \operatorname{Max}(T)$ such that $M=M_{1} \cap M_{2}$ and the natural maps $R / M \rightarrow$ $T / M_{1}$ and $R / M \rightarrow T / M_{2}$ are both isomorphisms.

(c) ramified case: there exists $M^{\prime} \in \operatorname{Max}(T)$ such that $M^{\prime 2} \subseteq M \subset M^{\prime},[T / M: R / M]=2$, and the natural map $R / M \rightarrow T / M^{\prime}$ is an isomorphism.

In each of the above three cases, $M$ is the crucial ideal of $R \subset T$.

In the context of Theorem 4 , consider the field $K$ := $R / M$. Recall (as in the proof of [9, Corollary II.2]) that the "decomposed" (resp., "ramified") case in Theorem 4 corresponds to $T / M T$ being isomorphic, as a $K$-algebra, to $K \times K$ (resp., to $\left.K[Y] /\left(Y^{2}\right)\right)$.

Lemma 5 (see [6, Proposition 4.6]). Let $f: A \hookrightarrow B$ be a ring extension. Then $f$ is a minimal extension if (and only if) there is a maximal ideal $M$ of $A$ such that the induced extension $f_{M}$ : $A_{M} \rightarrow B_{M}$ is minimal and $A_{N}=B_{N}$ for each prime ideal $N \neq$ $M$. Moreover, whenever these (equivalent) conditions hold, $M$ (resp., $M A_{M}$ ) is the crucial maximal ideal of $A \subset B$ (resp., $\left.A_{M} \subset B_{M}\right)$, and the minimal extensions $A \subset B$ and $A_{M} \subset B_{M}$ are of the same type.

The following result will be useful.

Theorem 6 (see [4, Theorem 3.4]). Let $f: R \hookrightarrow S$ be an extension. Then, the natural map $f^{\prime}: R(X) \hookrightarrow S(X)$ is a minimal extension if and only if $f$ is a minimal extension. If these (equivalent) conditions hold, then one has the following three conclusions.

(a) $R(X) \otimes_{R} S=S(X)$ canonically.

(b) $f$ and $f^{\prime}$ are the same type of minimal extension.

(c) If $M:=\mathscr{C}(R, S)$, then $\mathscr{C}(R(X), S(X))=M R(X)$.

The next two results recall/develop some facts about integral $t$-closed FIP extensions that will be used in Section 3.

Proposition 7. Let $R \subset S$ be an integral t-closed FIP extension. Then,

(1) there is a finite chain of minimal extensions, $R_{0} \subseteq \cdots \subseteq$ $R_{i} \subset R_{i+1} \subseteq \cdots \subseteq R_{n}$, in which $R_{0}=R, R_{n}=S$, and each $R_{i} \subset R_{i+1}$ is inert;

(2) the canonical map $\operatorname{Spec}(S) \rightarrow \operatorname{Spec}(R)$ is a homeomorphism (in the Zariski topology). Moreover, there is a positive integer $m$ such that $(R: S)$ is an intersection of $m$ pairwise distinct maximal ideals of $S$ and also an intersection of $m$ pairwise distinct maximal ideals of $R$.
Proof. (1) is a special case of [1, Lemma 5.6].

(2) Using (1), take $\left\{R_{i} \mid 0 \leq i \leq n\right\}$ to be a finite maximal chain of inert minimal extensions with $R_{0}:=R$ and $R_{n}:=S$. In view of Theorems 4 and $3(\mathrm{~d})$, the canonical continuous map $\operatorname{Spec}\left(R_{i+1}\right) \rightarrow \operatorname{Spec}\left(R_{i}\right)$ (which is a Zariskiclosed map, owing to integrality) is a bijection and hence a homeomorphism for all $0 \leq i \leq n-1$. By composing these maps, we see that the canonical map $\theta: \operatorname{Spec}(S) \rightarrow \operatorname{Spec}(R)$ is also a homeomorphism. Since any $t$-closed extension is seminormal, [1, Lemma 4.8] shows that $(R: S)$ is a radical ideal of $S$ (and hence also a radical ideal of $R$ ). Hence, by a characterization of integral FCP extensions in $[1$, Theorem 4.2(a)], $(R: S)$ is an intersection of finitely many, say, $m$, pairwise distinct maximal ideals of $S$. Since $\theta$ is a bijection and integrality ensures that $\theta^{-1}\left(V_{R}(R: S)\right)=V_{S}(R: S)$, it follows that $(R: S)$ is also an intersection of $m$ pairwise distinct maximal ideals of $R$.

Proposition 8 (see [10, Lemme 3.10]). Let $K$ be a field and let $K \subset R$ be an integral ring extension. Then, $K \subset R$ is $t$-closed if and only if $R$ is a field.

\section{T-Closed FIP Extensions of Nagata Rings}

Consider an FIP field extension $K \subseteq L$ and an indeterminate $X$. The first goal of this section is to show that the map $\varphi:[K, L] \rightarrow[K(X), L(X)]$ defined by $\varphi(T)=T(X)$ is an order-isomorphism. We will need to consider two cases, namely, where $|K|$ is finite and where $|K|$ is infinite. It will be convenient to use the following version of the Primitive Element Theorem: a finite-dimensional field extension $K \subseteq L$ has FIP if and only if $L=K[x]$ for some $x \in L$. (Note also that if $K \subseteq L$ is any FIP extension of fields, then $[L: K]<\infty$.)

Proposition 9. Let $K \subseteq L$ be an FIP field extension, where $K$ is a finite field. Then, the map $\varphi:[K, L] \rightarrow[K(X), L(X)]$, given by $T \mapsto T(X)$, is an order-isomorphism, and so $K(X) \subseteq$ $L(X)$ has FIP.

Proof. Since $K$ is a finite field and $[L: K]<\infty, L$ is a finitedimensional Galois extension of $K$ (cf. [11, Proposition 4, Ch. V, Sec. 12, p. 91], taking $N^{\prime}:=L(X)$ and $\left.K^{\prime}:=K(X)\right)$. Hence, $K(X) \subseteq L(X)$ is a Galois extension (cf. [11, Théorème 5, Ch. Vl, Sec. 10, p. 68]). Then [11, Corollaire 1, Ch. V, Sec. 10, p. 69] shows that for each $T^{\prime} \in[K(X), L(X)]$, there exists (a unique) $T \in[K, L]$ such that $T^{\prime}=T(X)$ and $T=T^{\prime} \cap L$. In particular, $\varphi$ is surjective and hence an order-isomorphism.

Before getting a result similar to Proposition 9 for the case of an infinite field, we need a lemma. It will use the following definition: if $K$ is a field and $F(X) \in K(X)$, let $F^{*}(K)$ denote the set of all $F(t) \in K$ such that $F(t)$ exists (for some $t \in K$ ).

Lemma 10. Let $K$ be an infinite field and $F(X) \in K(X)$ such that the set $F^{*}(K)$ is finite. Then, $F(X) \in K$.

Proof. Write $F(X)=P(X) / Q(X)$, where $P(X)$ and $Q(X)$ are two relatively prime polynomials in $K[X]$ and $Q(X) \neq 0$. Since the set of values $F^{*}(K)$ is finite and $K$ is infinite, we can 
see (by ignoring the finitely many roots of $Q(X)$ in $K$ ) that there must exist some value $a \in K$ that is attained infinitely often, that is, such that $\{t \in K \mid F(t)=a\}$ is infinite. But $a=F(t)=P(t) / Q(t)=a$, with $t \in K$ such that $Q(t) \neq 0$, gives $P(t)-a Q(t)=0$, so that $P(X)-a Q(X) \in K[X]$ has infinitely many roots in $K$. Thus, $P(X)-a Q(X)=0$, giving that $F(X)=P(X) / Q(X)=a \in K$.

Proposition 11. Let $K \subset L$ be an FIP field extension, where $K$ is an infinite field. Then, the map $\varphi:[K, L] \rightarrow[K(X), L(X)]$, given by $T \mapsto T(X)$, is an order-isomorphism, and so $K(X) \subseteq$ $L(X)$ has FIP.

Proof. By the Primitive Element Theorem, $L=K[\alpha]$ for some $\alpha \in L$. Let $P(Y) \in K[Y]$ denote the (monic) minimal polynomial of $\alpha$ over $K$. A standard proof of the Primitive Element Theorem (as given, for instance, in [11, Théorème 1, Ch. V, Sec. 7, p. 39]) shows that the $K$-subalgebras of $L$ are of the form $E_{Q}$, where $E_{Q}$ denotes the $K$-subalgebra of $L$ generated by the coefficients of $Q(Y)$, as $Q(Y)$ runs over the set of monic polynomials in $L[Y]$ that divide $P(Y)$ in $L[Y]$. (The reader is cautioned that the notation $E_{Q}$ does not refer to a ring of fractions but merely to a $K$-algebra that is constructed from $Q(Y)$ in a certain way.) We will show that each $K(X)$-subalgebra of $L(X)$ is of the form $E_{Q}(X)$ for some suitable $Q(Y)$.

Observe that $K(X) \subseteq L(X)$ has FIP since $L(X)=$ $(K(X))[\alpha]$; and $[L(X): K(X)]=\left[\begin{array}{lll}L & : & K\end{array}\right]$ because $L(X) \cong L \otimes_{K} K(X)$ (by, for instance, [11, Théorème 5, Ch. V, Sec. 10, p. 68] or [4, Lemma 3.1(e)]). Therefore, $P(Y) \in$ $(K(X))[Y]$ is also the minimal polynomial of $\alpha$ over $K(X)$. We next proceed to describe the $K(X)$-subalgebras of $L(X)$ by reapplying the method that was used above to describe the $K$-subalgebras of $L$.

Let $B(Y), D(Y) \in(L(X))[Y]$ be two monic polynomials such that $P(Y)=B(Y) D(Y)$. Write $P(Y):=\sum a_{i} Y^{i}, B(Y):=$ $\sum b_{i}(X) Y^{i}$, and $D(Y):=\sum d_{i}(X) Y^{i}$, where $a_{i} \in K$ and $b_{i}(X), d_{i}(X) \in L(X)$ for each $i$, so that we have the following equation: $\sum a_{i} Y^{i}=\left(\sum b_{i}(X) Y^{i}\right)\left(\sum d_{i}(X) Y^{i}\right)(*)$. For a fixed $t \in L$, consider the substitution $X \mapsto t$. Then, $(*)$ gives $P(Y)=$ $\sum a_{i} Y^{i}=\left(\sum b_{i}(t) Y^{i}\right)\left(\sum d_{i}(t) Y^{i}\right)$, supposing for the moment that all the expressions $b_{i}(t)$ and $d_{i}(t)$ are meaningful. Under this assumption, it would follow that $\sum b_{i}(t) Y^{i}$ and $\sum d_{i}(t) Y^{i}$ each divide $P(Y)$ in $L[Y]$ for each $t \in L$. As there are only finitely many such monic polynomials, it must be the case that for each $i$, the sets $b_{i}^{*}(L)$ and $d_{i}^{*}(L)$ are each finite. Since $L$ is an infinite field, it follows from Lemma 10 that for each $i$, we have $b_{i}(X), d_{i}(X) \in L$. Consequently, $B(Y)$ and $D(Y)$ each divide $P(Y)$ in $L[Y]$. Hence, the $K(X)$-subalgebras of $L(X)$ are of the form $E_{Q}^{\prime}$, where $E_{Q}^{\prime}$ denotes the $K(X)$-subalgebra of $L(X)$ generated by the coefficients of $Q(Y)$, as $Q(Y)$ runs over the set of monic polynomials in $L[Y]$ that divide $P(Y)$ in $L[Y]$. It follows that $E_{Q}^{\prime}=E_{Q}(X)$, where, as above, $E_{Q}$ denotes the $K$-subalgebra of $L$ generated by the coefficients of $Q(Y)$. In particular, $\varphi$ is surjective and hence an order-isomorphism, as asserted.

In the context of the preceding proof, it is interesting to note that since $K \subseteq E_{\mathrm{Q}}$ has FIP, we can write $E_{\mathrm{Q}}=K\left[\alpha_{\mathrm{Q}}\right]$ for some $\alpha_{\mathrm{Q}} \in L$. Then, $E_{\mathrm{Q}}^{\prime}=E_{\mathrm{Q}}(X)=K(X)\left[\alpha_{\mathrm{Q}}\right]$. Thus, not only does $\alpha_{\mathrm{Q}}$ generate $E_{\mathrm{Q}}$ over $K$, but it also generates $E_{\mathrm{Q}}^{\prime}$ over $K(X)$.

We can now present this paper's first contribution to the question under consideration.

Theorem 12. Let $R \subset S$ be an integral $t$-closed FIP extension.

Then, $|[R(X), S(X)]|=|[R, S]|$, the function $\varphi:[R, S] \rightarrow$ $[R(X), S(X)]$ is an order-isomorphism, and $R(X) \subseteq S(X)$ is an FIP extension.

Proof. Since $\varphi$ is an order-preserving and order-reflecting injection, it suffices to prove the first assertion. As $R \subseteq S$ has FIP, we have $|\operatorname{Supp}(S / R)|<\infty$, by [1, Corollary 3.2]. Set $n:=|\operatorname{MSupp}(S / R)|$ and write $\operatorname{MSupp}(S / R)=:\left\{M_{1}, \ldots, M_{n}\right\}$. By [1, Theorem 3.6], the map $\psi:[R, S] \rightarrow \prod_{i=1}^{n}\left[R_{M_{i}}, S_{M_{i}}\right]$ defined by $\psi(T)=\left(T_{M_{1}}, \ldots, T_{M_{n}}\right)$ is a bijection and $\mid[R$, $S]\left|=\prod_{i=1}^{n}\right|\left[R_{M_{i}}, S_{M_{i}}\right] \mid$. In the same way, we can show that $|[R(X), S(X)]|=\prod_{i=1}^{n}\left|\left[R_{M_{i}}(X), S_{M_{i}}(X)\right]\right|$, because $R_{M}(X)=$ $(R(X))_{M R(X)}$ and $S_{M}(X)=(S(X))_{M R(X)}$ for each $M \in \operatorname{Max}(R)$ and, by [4, Lemma 3.3], $\operatorname{MSupp}(S(X) / R(X))=\{M R(X) \mid$ $M \in \operatorname{MSupp}(S / R)\}$. (Note that when we applied [1, Theorem 3.6] to $R(X) \subset S(X)$, we did not need to know already that this extension has FIP; it was enough that this extension has FCP, which it indeed inherits from $R \subset S$ by [4, Theorem 3.9].) Thus, if $\left|\left[R_{M_{i}}, S_{M_{i}}\right]\right|=\left|\left[R_{M_{i}}(X), S_{M_{i}}(X)\right]\right|$ for each $i \epsilon$ $\{1, \ldots, n\}$, then $|[R, S]|=|[R(X), S(X)]|$. So, without loss of generality, we may assume that $(R, M)$ is a quasi-local ring which is properly contained in $S$. Note that, in passing from $R \subset S$ to $R_{M_{i}} \subset S_{M_{i}}$, the extension has retained the "integral $t$-closed FIP" hypothesis. Therefore, [4, Lemma 3.17] can be applied, giving that $\operatorname{Max}(S)=\{M\}$; necessarily, $M=(R$ : $S)$. Thus, by a standard homomorphism theorem, $|[R, S]|=$ $|[R / M, S / M]| ;$ similarly, as $M R(X)=(R(X): S(X))$, we get

$$
|[R(X), S(X)]|=\left|\left[\frac{R(X)}{M R(X)}, \frac{S(X)}{M R(X)}\right]\right|
$$

(cf. also [1, Proposition 3.7(c)]).

Since $|[R(X) / M R(X), S(X) / M R(X)]|=\mid[(R / M)(X),(S /$ $M)(X)] \mid$ and it follows from Propositions 9 and 11 that the FIP field extension $R / M \subset S / M$ satisfies $|[R / M, S / M]|=$ $|[(R / M)(X),(S / M)(X)]|$, the equalities that we have collected combine to show that $|[R, S]|=|[R / M, S / M]|=$ $|[R(X), S(X)]|$. The proof is complete.

We close this section with some comments about Galois groups and Galois extensions of rings, some of which will be used in the next section. In particular, the isomorphism in Lemma 13 will play a key role in the proof of Lemma 25.

Lemma 13. Let $K \subseteq T$ be an algebraic field extension and let $\Gamma$ (resp., $\left.\Gamma^{\prime}\right)$ be the group of $K$-automorphisms (resp., $K(X)$-automorphisms) of $T$ (resp., of $T(X))$. Then there is an isomorphism $\pi: \Gamma \rightarrow \Gamma^{\prime}$, denoted by $\pi(\sigma)=\sigma^{\prime}$, such that $\sigma^{\prime}\left(\sum a_{i} X^{i}\right)=\sum \sigma\left(a_{i}\right) X^{i}$ for each $\sigma \in \Gamma$ and each $\sum a_{i} X^{i} \epsilon$ $T[X]$. Moreover, the canonical map $K(X) \otimes_{K} T \rightarrow T(X)$ is an isomorphism. 
Proof. Let $\sigma \in \Gamma$ and $f(X)=\left(\sum a_{i} X^{i}\right) /\left(\sum b_{i} X^{i}\right) \in T(X)$. It is easy to check that we can well define a function $\sigma^{\prime}: T(X) \rightarrow$ $T(X)$ by $\sigma^{\prime}(f(X)):=\left(\sum \sigma\left(a_{i}\right) X^{i}\right) /\left(\sum \sigma\left(b_{i}\right) X^{i}\right) \in T(X)$. It is then clear that $\sigma^{\prime} \in \Gamma^{\prime}$.

Conversely, for each $\sigma^{\prime} \in \Gamma^{\prime}$, let $\sigma$ denote the restriction of $\sigma^{\prime}$ to $T$. Since $T$ is algebraic over $K$ and algebraically closed in $T(X)$, it is easy to see that $\sigma$ maps $T$ (injectively) into itself. (Similarly, so does the restriction of $\sigma^{\prime-1}$ to T.) This mapping is, in fact, surjective, for if $t \in T$ and we take $g \in T(X)$ such that $\sigma^{\prime}(g)=t$, then $g=\sigma^{\prime-1}(t) \in T$. Consequently, $\sigma \in \Gamma$.

It is now easy to check that the function $\pi: \Gamma \rightarrow \Gamma^{\prime}$ defined by $\pi(\sigma)=\sigma^{\prime}$ is an isomorphism. The final assertion is a special case of $[4, \operatorname{Lemma} 3 \cdot 1(\mathrm{e})]$.

Recall that there is a theory of Galois ring extensions that generalizes the theory of (finite-dimensional) Galois field extensions. A summary of much of that theory appears in Section 1 of a book by Greither [12], with which we will assume familiarity. One may also find many examples in that book. For an extension of rings $R \subseteq S$, let $[R, S]^{s}$ denote the set of all $R$-subextensions $T$ of $S$ such that $R \subseteq T$ is separable (in the usual sense, namely, that $T$ is projective over $T \otimes_{R} T$ ). Recall also that a ring $R$ is said to be connected if its only idempotent elements are 0 and 1 . A ring $R$ is connected (if and) only if $R(X)$ is connected. Indeed, it was shown in [13, Theorem 2.4] that for any ring $A$, each idempotent element of $A(X)$ must belong to $A$.

Proposition 14. Let $R \subseteq S$ be a Galois extension of rings with finite Galois group $G$. Then the following assertions hold.

(1) $R(X) \subseteq S(X)$ is a Galois (ring) extension with Galois group isomorphic to $G$.

(2) If, in addition, $S$ is connected, then the canonical map $[R, S]^{s} \rightarrow[R(X), S(X)]^{s}$, given by $T \mapsto T(X)$, is an order-isomorphism.

Proof. (1) Since $R \subseteq S$ is integral (because Galois extensions with finite Galois groups are module-finite), [4, Lemma 3.1(e)] may be applied to show that the natural map $R(X) \otimes_{R} S \rightarrow S(X)$ is an isomorphism. Next, an application of $[12$, Lemma 1.11] shows that $R(X) \subseteq S(X)$ is a Galois extension with Galois group $G$.

(2) As $S$ is connected, the Chase-Harrison-Rosenberg Theorem tells us that $[R, S]^{s}$ has the same (finite) cardinality as the set of all subgroups of $G$ (cf. [12, Theorem 2.2]). Also, we noted above that $S(X)$ inherits the "connected" property from $S$. Thus, in view of (1), we see similarly that $[R(X), S(X)]^{s}$ also has the same cardinality as the set of all subgroups of $G$. It therefore suffices to show that if $T \in[R, S]^{s}$, then $T(X) \in[R(X), S(X)]^{s}$ (for then, the restriction of $\varphi$ to $[R, S]^{s} \rightarrow[R(X), S(X)]^{s}$ is a necessarily injective function, and an application of the Pigeon-hole Principle would finish the proof). In fact, $T$ inherits from $S$ the property of being integral over $R$, and so, by another application of [4, Lemma 3.1(e)], $R(X) \otimes_{R} T=T(X)$ canonically. Since $T$ is separable over $R$ and separability is preserved by arbitrary base changes, it follows that $T(X) \in[R(X), S(X)]^{s}$, as desired.
Note that the preceding result gives another proof of the special case of Lemma 13 where $K \subseteq T$ is a finite-dimensional Galois field extension.

\section{The General Case}

The aim of this section is to prove that for any ring extension $R \subseteq S$ such that $R(X) \subseteq S(X)$ has FIP, the map $\varphi$ : $[R, S] \rightarrow[R(X), S(X)]$, given by $T \mapsto T(X)$, is an orderisomorphism. Since $\varphi$ is known to be an order-preserving and order-reflecting injection [4, Lemma 3.1(d)], it remains only to show that $\varphi$ is surjective. As an FIP extension has FCP, we will prove this result by induction on the length of a maximal chain of minimal extensions. That induction will begin in Proposition 31 by showing that if $R(X) \subseteq S(X)$ has FIP and $T^{\prime} \in[R(X), S(X)]$ is such that $R(X) \subset T^{\prime}$ is a minimal extension, there exists $T \in[R(X), S(X)]$ such that $T^{\prime}=T(X)$. We will need to first treat the case of a quasi-local base ring in Proposition 30, for which the following lemmas will be useful.

Lemma 15. Let $K$ be a field, with $K \subset R$ and $K \subset T$ minimal (ring) extensions such that the composite $S:=R T$ exists. If $K \subset$ $R$ is ramified and $K \subset T$ is decomposed, then $T$ is the only $T^{\prime} \in$ $[K, S]$ such that $K \subset T^{\prime}$ is a decomposed minimal extension satisfying $S=R T^{\prime}$.

Proof. Since $K \subset R$ is ramified, the comments following Theorem 4 provide an element $x \in R$ such that $R=K[x]$ and $x^{2}=0$ so that $R$ is a local ring with maximal ideal $M:=K x$. Since $K \subset T$ is decomposed, those same comments provide an element $y \in T$ such that $T=K[y]$ and $y^{2}=y$ so that $T$ has exactly two maximal ideals, say, $M_{1}:=K y$ and $M_{2}:=K(y-1)$. As $K$ is a field, the extensions $K \subset R$ and $K \subset T$ necessarily have the same crucial maximal ideal (i.e., $\{0\}$ ), and so [6, Proposition 7.6] can be applied. There are three cases.

(1) Assume that $M M_{1}=0$. Then, by [6, Proposition 7.6(a)], $R \subset S$ is a decomposed minimal extension and $T \subset S$ is a ramified minimal extension. Since $M M_{1}=0$, we have $x y=0$, and so $S=K[x] K[y]=(K+K x)(K+K y)=$ $K+K x+K y$. Thus, $\{1, x, y\}$ is a $K$-basis of $S$. We show next that, given $R$ and $S$, if $T^{\prime \prime} \in[K, S]$ is such that $K \subset T^{\prime \prime}$ is a decomposed minimal extension with $S=R T^{\prime \prime}$, then $T^{\prime \prime}=T$. As above, we can write $T^{\prime \prime}=K\left[y^{\prime \prime}\right]$, for some $y^{\prime \prime} \in T^{\prime \prime}$ such that $y^{\prime \prime 2}=y^{\prime \prime}$. Write $y^{\prime \prime}:=a+b x+c y$, for some $a, b, c \in K$. As $y^{\prime \prime 2}=y^{\prime \prime}$, we get $a^{2}+c^{2} y+2 a b x+2 a c y=a+b x+c y$ so that $a^{2}=a(*)$ and $(2 a-1) b=0(* *)$. By $(*)$, either $a=1$ or $a=0$. Thus, by $(* *), b=0$ in any event. Hence, $y^{\prime \prime}$ is either $1+c y$ or $c y$, and so $y^{\prime \prime} \in T$. Then $K \subset T^{\prime \prime} \subseteq T$, whence $T^{\prime \prime}=T$ by the minimality of $K \subset T$.

(2) Assume that $M M_{2}=0$. Then one can reason as in case (1), with $y-1$ replacing $y$.

(3) Finally, assume that $M M_{1} \neq 0 \neq M M_{2}$. By the assumptions of (3), the elements $x$ and $y$ that were introduced above satisfy $x y \neq 0$ and $x(y-1) \neq 0$. Notice that $x$ and $x y$ are linearly independent over $K$. (Otherwise, $x=\beta x y$ for some $\beta \in K$; multiplication by $y$ leads to $x y=\beta x y$, 
whence $\beta=1$ and $x=x y$, a contradiction.) In fact, we claim that $x y$ is not in the $K$-span of $\{1, x, y\}$. If the claim fails, $x y=\lambda+\mu x+v y$ for some $\lambda, \mu, v \in K$. Multiplication by $x$ leads to $0=\lambda x+v x y$. Then, by the above comment about linear independence, $\lambda=0=\nu$, whence $x y=\mu x$, contradicting that same comment. This proves the claim. Hence, $\{1, x, y, x y\}$ is a $K$-basis of $(K+K x)(K+K y)=R T=S$. We proceed to prove that, given $R$ and $S$, if $T^{\prime \prime} \in[R, S]$ is such that $K \subset T^{\prime \prime}$ is a decomposed minimal extension with $S=R T^{\prime \prime}$, then $T^{\prime \prime}=T$.

As $T^{\prime \prime}=K\left[y^{\prime \prime}\right]$ for some element $y^{\prime \prime}=y^{\prime \prime 2}$, we have $S=R T^{\prime \prime}=(K+K x)\left(K+K y^{\prime \prime}\right)=K+K x+K y^{\prime \prime}+K x y^{\prime \prime}$. Write $y^{\prime \prime}:=a+b x+c y+d x y$, with $a, b, c, d \in K$. As $y^{\prime \prime 2}=y^{\prime \prime}$, we get $a^{2}+c^{2} y+2 a b x+2 a c y+2 a d x y+2 b c x y+2 c d x y=$ $a+b x+c y+d x y$ so that $a^{2}=a(*),(2 a-1) b=0(* *), c(c+$ $2 a-1)=0(* * *)$ and $2 a d+2 b c+2 c d-d=0(* * * *)$. By (*), either $a=1$ or $a=0$, and so by $(* *), b=0$ in any event.

Suppose first that $a=1$. Then $(* * *)$ gives $c(c+1)=0(\dagger)$ and $(* * * *)$ gives $d(1+2 c)=0(\dagger \dagger)$. From $(\dagger)$ and $(\dagger \dagger)$, we deduce that either $c=d=0$ (in which case, $y^{\prime \prime}=a=1 \in K$ and $T^{\prime \prime}=K$, a contradiction) or $c=-1$ and $d=0$ (in which case, $y^{\prime \prime}=1-y \in T$, and so the minimality of $K \subset T$ forces $T^{\prime \prime}=T$, as desired).

Lastly, suppose that $a=0$. Then $(* * *)$ gives $c(c-1)=$ $0(\ddagger)$ and $(* * * *)$ gives $d(-1+2 c)=0(\ddagger \ddagger)$. Combining $(\ddagger)$ and ( $\ddagger$ ), we get either $c=d=0$ (in which case, $y^{\prime \prime}=0 \in K$ and $T^{\prime \prime}=K$, a contradiction) or $c=1$ and $d=0$ (in which case, $y^{\prime \prime}=y \in T$, and so the minimality of $K \subset T$ forces $\left.T^{\prime \prime}=T\right)$.

Proposition 16. Let $R \subset T_{1}$ and $R \subset T_{2}$ be minimal ring extensions of a quasi-local ring $(R, M)$, such that the composite $S:=T_{1} T_{2}$ exists and such that $R \subset T_{1}$ is ramified and $R \subset T_{2}$ is decomposed. Then $T_{2}$ is the only $T \in[R, S]$ such that $R \subset T$ is a decomposed minimal extension satisfying $T_{1} T=S$.

Proof. Since $R \subset T_{1}$ and $R \subset T_{2}$ are integral minimal extensions, we get that (the crucial maximal ideal) $M$ is a common ideal of $R, T_{1}$, and $T_{2}$ and hence also an ideal of $S$. Put $K:=R / M, T_{1}^{\prime}:=T_{1} / M, T_{2}^{\prime}:=T_{2} / M$, and $S^{\prime}:=S / M$. We are reduced to the situation of Lemma 15 , since $K \subset T_{i}^{\prime}$ is a minimal extension of the same type as $R \subset T_{i}$, for $i=1,2$. Hence, by Lemma $15, T_{2}^{\prime}$ is the only ring $E \in\left[K, S^{\prime}\right]$ such that $K \subset E$ is a decomposed minimal extension satisfying $S^{\prime}=T_{1}^{\prime} E$. Now, suppose that $T \in[R, S]$ is such that $R \subset T$ is a decomposed minimal extension satisfying $T_{1} T=S$. Then $T / M \in[K, S / M]$ is such that $K \subset T / M$ is a decomposed minimal extension and $T_{1}^{\prime}(T / M)=S^{\prime}$, and so $T / M=$ $T_{2}^{\prime}\left(=T_{2} / M\right)$. With $\varphi: S \rightarrow S / M$ denoting the canonical surjection, it follows that $T=\varphi^{-1}(T / M)=\varphi^{-1}\left(T_{2}^{\prime}\right)=T_{2}$.

The next lemma uses the notion of the ideal-length $\lambda_{R}(I)$ of an ideal $I$ of a ring $R$, in the sense of [14, Definition, p. 233]. For the sake of completeness, we recall that definition: $\lambda_{R}(I):=\ell\left(R_{\mathfrak{K}} / I R_{\mathfrak{K}}\right)$, where $\ell$ denotes the length of an $R$-module (with such length taken to be $\infty$ if the module does not have a composition series) and $\mathfrak{\Omega}$ denotes the complement of the union of the associated primes of $I$.
To motivate Lemma 17, note that the following is a consequence of the classification of the minimal extensions of a field [3, Lemme 1.2]. If $K$ is a field and $K \subset S$ is a minimal (hence integral FIP) extension, then $S$ is not a reduced ring if and only if $S \cong K[Y] /\left(Y^{2}\right)$. In view of the comment following Theorem 4, the preceding assertion is the special case of Lemma 17 in which $R$ is a field and $R \subset S$ is a minimal extension.

Lemma 17. Let $R \subset S$ be an integral FIP extension, $C:=(R$ : $S)$, and $P \in V_{R}(C)$. Then $P S$ is not a radical ideal of $S$ if and only if there exists $T \in[R, S]$ such that $T \subset S$ is a ramified minimal extension such that $P \subseteq(T: S)$.

Proof. Since $R \subset S$ is an integral extension with FCP, [1, Theorem 4.2(a)] shows that both $R / C$ and $S / C$ are Artinian rings and hence of (Krull) dimension 0. In particular, $P \in$ $\operatorname{Max}(R)$. Set $R^{\prime}:=R / C, S^{\prime}:=S / C, P^{\prime}:=P / C$, and $M_{i}^{\prime}:=$ $M_{i} / C$ for each $M_{i} \in \operatorname{Spec}(S)$ that lies over $P$ in $R$; necessarily, $M_{i} \in V_{S}(C) \subseteq \operatorname{Max}(S)$.

Suppose first that there exists $T \in[R, S]$ such that $T \subset S$ is a minimal ramified extension with $P \subseteq Q:=(T: S)$. By Theorem 4(c), there exists $M \in \operatorname{Max}(S)$ with $M^{2} \subseteq$ $Q \subset M$. Consequently, $Q$ is an $M$-primary ideal of $S$. We proceed to derive a contradiction from the assumption that $P S$ is a radical ideal of $S$. Note that $S / P S \cong(S / C) /(P S / C)$ is an Artinian ring and, hence, has only finitely many prime (necessarily maximal) ideals. Thus, since $P S$ is being assumed radical, we have $P S=\cap_{k=1}^{d} M_{k}$, where $M_{1}=M, \ldots, M_{d}$ is the (finite) list of (pairwise distinct) maximal ideals of $S$ that contain PS. Therefore, by the Chinese Remainder Theorem, $S / P S \cong \prod_{k=1}^{d} S / M_{k}$, a direct product of finitely many fields. Thus, $S / Q \cong(S / P S) /(Q / P S)$ is also isomorphic to a direct product of finitely many fields and hence is a reduced ring. So $Q$ is a radical ideal of $S$. But the radical of $Q$ in $S$ is $M$. Since $M \neq Q$, we have the desired contradiction.

For the converse, assume that $P S$ is not a radical ideal of $S$; equivalently, $P^{\prime} S^{\prime}$ is not a radical ideal of $S^{\prime}$. As $S^{\prime}$ is zero-dimensional and Noetherian (i.e., Artinian), a classic result [14, Theorem 9, page 213] gives a unique primary decomposition of $P^{\prime} S^{\prime}$ in $S^{\prime}$, namely, $P^{\prime} S^{\prime}:=\cap_{j=1}^{n} Q_{j}^{\prime}$, where $Q_{j}^{\prime}$ is a $P_{j}^{\prime}$-primary ideal of $S^{\prime}$ for each $j$. As $P^{\prime} S^{\prime}$ is not a radical ideal of $S^{\prime}$, there exists an index $i$ such that $Q_{i}^{\prime} \subset P_{i}^{\prime}$. Fix $i$.

As $S^{\prime}$ is zero-dimensional, no ideal of $S^{\prime}$ has embedded components. Hence, by [14, Theorem 24, p. 234 and Theorem 26, p. 235], $Q_{i}^{\prime}$ is a $P_{i}^{\prime}$-primary ideal of finite ideal-length, with $1 \leq \lambda_{S^{\prime}}\left(Q_{i}^{\prime}\right)<\infty$; moreover, there exists a $P_{i}^{\prime}$-primary ideal $Q_{i}^{\prime \prime}$ of $S^{\prime}$ such that $Q_{i}^{\prime} \subseteq Q_{i}^{\prime \prime} \subset P_{i}^{\prime}$, with $Q_{i}^{\prime \prime}$ and $P_{i}^{\prime}$ adjacent ideals. Thus, $P_{i}^{\prime 2} \subseteq Q_{i}^{\prime \prime}$ by [14, Corollary 2, p. 237]. It follows that $S^{\prime} / Q_{i}^{\prime \prime}$ is a quasi-local Artinian ring with maximal ideal $P_{i}^{\prime} / Q_{i}^{\prime \prime}$ such that $\left(P_{i}^{\prime} / Q_{i}^{\prime \prime}\right)^{2}=0$.

Consider the canonical surjection $f: S \rightarrow S / C$. Put $P_{i}:=f^{-1}\left(P_{i}^{\prime}\right)$ and $Q_{i}:=f^{-1}\left(Q_{i}^{\prime \prime}\right)$. Note that $P_{i} \in \operatorname{Max}(S)$ and $Q_{i}$ is a $P_{i}$-primary ideal of $S$. By a standard homomorphism theorem, the above "adjacency" assertion implies that $Q_{i}$ and $P_{i}$ are adjacent ideals of $S$; and $S / Q_{i} \cong S^{\prime} / Q_{i}^{\prime \prime}$ is a quasi-local 
Artinian ring with maximal ideal $\left.P_{i} / Q_{i} \cong P_{i}^{\prime} / Q_{i}^{\prime \prime}\right)$ such that $\left(P_{i} / Q_{i}\right)^{2}=0$.

We have $P \subseteq P S \subseteq Q_{i}$ so that $P=Q_{i} \cap R$ and the field $R / P$ can now be viewed as a subring of $S / Q_{i}$. We next apply a piece of the structure theory of complete local rings. By the proof of $\left[15\right.$, Corollaire 19.8 .10$, p. 113], $S / Q_{i} \cong$ $\left(\left(S / P_{i}\right)\left[\left[T_{1}, \ldots, T_{m}\right]\right]\right) / N$, where $N:=\left(T_{1}, \ldots, T_{m}\right)^{2}$ and $m$ is the vector-space dimension of $P_{i} / P_{i}^{2}$ over $S / P_{i}$. It follows that $S / Q_{i}$ has a field of representatives $K \cong S / P_{i}$ which contains $R / P$.

Let $\psi: S \rightarrow S / Q_{i}$ be the canonical surjection and $\operatorname{set} T:=$ $\psi^{-1}(K)$ so that $Q_{i}$ is an ideal of $T$ satisfying $T / Q_{i} \cong \psi(T)=$ $K \cong S / P_{i}$. It follows that $Q_{i} \in \operatorname{Max}(T)$. Also, recall that $P_{i}^{2} \subseteq Q_{i}$. Thus, if we wish to establish that $T \subset S$ is a minimal ramified extension with $P \subseteq Q_{i}=(T: S)$ by appealing to Theorem 4(c), it suffices to prove that $\left[S / Q_{i}: T / Q_{i}\right]=2$. To that end, note first that $\left(S / Q_{i}\right) /\left(P_{i} / Q_{i}\right) \cong S / P_{i} \cong T / Q_{i}$ is a one-dimensional vector space over $T / Q_{i}$. But $P_{i} / Q_{i}$ is also a one-dimensional vector space over $S / P_{i}\left(\cong T / Q_{i}\right)$ because $Q_{i}$ and $P_{i}$ are adjacent ideals of $S$. This completes the proof.

We can now give the first and second of the results in this section wherein a suitable ring in $[R(X), S(X)]$ is shown to take the desired $T(X)$ form.

Proposition 18. Let $(R, M)$ be a quasi-local ring, $R \subset S$ a ramified minimal extension, and $S \subset U$ a decomposed minimal extension. Let $T^{\prime} \in[R(X), U(X)]$ be such that $T^{\prime} \subset U(X)$ is a ramified minimal extension. Then there exists $T \in[R, U]$ such that $T^{\prime}=T(X)$.

Proof. We know that $(R(X), M R(X))$ is a quasi-local ring and that $U$ and $U(X)$ each have exactly two maximal ideals. We claim that the integral extension $R \subset U$ has FIP. To see this, note first that $R \subset S$ is subintegral (since it is ramified) and $S \subset U$ is seminormal (since it is decomposed: cf. [1, Lemma 5.3(a)]). Therefore, $S={ }_{U}^{+} R$. As $R \subset{ }_{U}^{+} R$ and ${ }_{U}^{+} R \subset U$ necessarily each have FIP (being minimal extensions) and $R \subset U$ is infra-integral (as a consequence of parts (b) and (c) of Theorem 4), it therefore follows from [1, Proposition 5.5] that $R \subset U$ has FIP, thus proving the above claim. Hence, by Lemma $17, M U(X)$ is not a radical ideal of $U(X)$.

We next claim that $M U$ is not a radical ideal of $U$. Since $R \subset S$ is ramified and $S \subset U$ is decomposed, it follows from Theorem 4 (and integrality) that there are exactly two prime ideals of $U$ lying over $M$. Denote these prime ideals by $M_{1}$ and $M_{2}$, and note that $\left\{M_{1}, M_{2}\right\}=\operatorname{Max}(U)$. Thus, $U(X)$ has exactly two maximal ideals, namely, $M_{1} U(X)$ and $M_{2} U(X)$. Suppose that the claim fails; that is, $M U$ is a radical ideal of $U$. Then $M U$ is an intersection of some prime ideals of $U$, and each of these primes must be maximal (because it lies over the maximal ideal $M)$. Thus, either $M U=M_{1} \cap M_{2}$ or $M U$ is of the form $M_{i}$. Hence, $M U(X)$ is either $M_{1} U(X) \cap M_{2} U(X)$ or of the form $M_{i}(X)$. Thus, $M U(X)$ is a radical ideal of $U$, the desired contradiction, thus proving the above claim. Hence, by another application of Lemma 17, there exists $T \in[R, U]$ such that $T \subset U$ is a ramified minimal extension.

Consider $I:=\mathscr{C}(S, U)=(S: U)$ and $J:=\mathscr{C}(T, U)=$ $(T: U)$. We claim that $I$ and $J$ are incomparable ideals of $U$.
To see this, first observe that $I$ is the intersection of the two maximal ideals of $U$, while Theorem 4(c) ensures that $J$ is a primary nonmaximal ideal of $U$ whose radical is a maximal ideal of $U$. It is now clear that $J \nsubseteq I$. On the other hand, if $I \subset J$, then $U / I$ (which, by the Chinese Remainder Theorem, is isomorphic to a direct product of two fields) would map homomorphically onto $U / J$, which is a nonzero quasi-local ring but not a field. This contradiction establishes the above claim. Thus, [6, Proposition 6.6(a)] can be applied to the base ring $T \cap S$. It follows that $T \cap S \subset S$ is a minimal extension. Thus, the minimality of $R \subset S$ gives $T \cap S=R$, and then [6, Proposition 6.6(a)] shows that $R \subset T$ inherits the "decomposed minimal extension" property from $S \subset U$. Then, by Theorem $6, R(X) \subset T(X)$ is also a decomposed minimal extension.

It remains only to prove that $T^{\prime}=T(X)$. This, in turn, is a consequence of the uniqueness assertion in Proposition 16. To check the applicability of that result here, it suffices to note that $S(X) T(X)=S(X) T^{\prime}$. Notice that $R(X) \subset U(X)$ can be obtained by "composing" the ramified minimal extension $R(X) \subset S(X)$ and the decomposed minimal extension $S(X) \subset U(X)$. As $R(X) \subset U(X)$ is therefore infra-integral, it follows from [1, Lemma 5.4] that each maximal chain of rings going from $R(X)$ to $U(X)$ has length 2 . Therefore, the integral extension $R(X) \subset T^{\prime}$ must be a minimal extension, necessarily decomposed since two distinct prime ideals of $U(X)$ lie over $M$ in $R$. Next, since $S \nsupseteq T$, the minimality of $S \subset$ $U$ gives that $S T=U$, and so $S(X) T(X)=(S T)(X)=U(X)$. It therefore suffices to prove that $S(X) T^{\prime}=U(X)$. This, in turn, follows from the minimality of $T^{\prime} \subset U(X)$, since $T^{\prime} \nsupseteq S(X)$. The proof is complete.

Proposition 19. Let $(R, M)$ be a quasi-local ring and let $R \subset S$ be an extension such that $R(X) \subset S(X)$ is an FIP extension with $R \subset{ }_{S}^{+} R$. Let $T^{\prime} \in[R(X), S(X)]$ be such that $R(X) \subset T^{\prime}$ is a decomposed minimal extension. Then there exists $T \in[R, S]$ such that $T^{\prime}=T(X)$.

Proof. Set $S_{1}:={ }_{S}^{+} R$ and $S_{2}:={ }_{S}^{t} R$. Then $S_{1} \subseteq S_{2}, S_{1}(X)=$ $\stackrel{+}{S(X)} R(X)$, and $S_{2}(X)={ }_{S(X)}^{t} R(X)$ by [4, Lemma 3.15]. As $R(X) \subset T^{\prime}$ is infra-integral, it follows that $T^{\prime} \subseteq S_{2}(X)$. Since $R(X) \subset S(X)$ is an FIP extension, each maximal chain of rings going from $R(X)$ to $S(X)$ must be finite. Set $R_{0}:=R$ and $T_{0}^{\prime}:=T^{\prime}$. We will inductively construct two increasing chains $\left\{R_{j}(X)\right\} \subseteq\left[R(X), S_{1}(X)\right]$ and $\left\{T_{j}^{\prime}\right\} \subseteq\left[T^{\prime}, S_{2}(X)\right]$, with $j \in$ $\{0, \ldots, 2 n\}$, for some integer $n$, such that $R_{2 n}(X)=S_{1}(X)$ and also such that the following induction hypothesis is satisfied for each $i \leq k \in\{1, \ldots, n\}: R_{2 i}(X) \subset T_{2 i}^{\prime}$ is a decomposed minimal extension and either $R_{2 i-1}(X) \subset R_{2 i}(X)$ and $T_{2 i-1}^{\prime} \subset$ $T_{2 i}^{\prime}$ are both ramified minimal extensions or we have both $R_{2 i-1}(X)=R_{2 i}(X)$ and $T_{2 i-1}^{\prime}=T_{2 i}^{\prime}$.

We begin with the induction basis, that is, the case $k=1$. As $R \subset S_{1}$, we can choose $R_{1} \in\left[R, S_{1}\right]$ such that $R \subset$ $R_{1}$ is a ramified minimal extension. Consider the two cases identified in [6, Proposition 7.6] corresponding to the choices $A:=R(X), B:=R_{1}(X)$, and $C:=T^{\prime}$ (along with $D:=B C$ ). In the first case, the induction hypothesis holds for $k=1$ if we take $R_{2}:=R_{1}$ and $T_{1}^{\prime}:=T_{2}^{\prime}:=D\left(=R_{1}(X) T^{\prime}\right)$. 
In the second case, [6, Proposition 7.6] provides certain rings $B^{\prime} \in[B, D]$ and $C^{\prime} \in[C, D]$. If we could find $R_{2} \in\left[R, S_{1}\right]$ such that $B^{\prime}=R_{2}(X)$ in this second case, then the induction hypothesis would hold for $k=1$ if we take $T_{1}^{\prime}:=C^{\prime}$ and $T_{2}^{\prime}:=$ $D\left(=R_{1}(X) T^{\prime}\right)$. To that end, it suffices, by [4, Theorem 3.4], to show that $B^{\prime}$ is contained in $S_{1}(X)$, the seminormalization of $R(X)$ in $S(X)$. As $B \subset B^{\prime}$ is a ramified minimal extension by [6, Proposition 7.6], it follows that $R(X) \subset B^{\prime}$ is subintegral, whence $B^{\prime} \subseteq S_{1}(X)$, thus completing the proof of the induction basis.

Next, for the induction step, suppose that the induction hypothesis holds for some $k \geq 1$. If $R_{2 k}(X)=S_{1}(X)$, the inductive construction of the chains of rings is terminated. Assume, instead, that $R_{2 k}(X) \neq S_{1}(X)$. We will sketch how to adapt the argument that was given for the induction basis. First, choose $R_{2 k+1} \in\left[R_{2 k}, S_{1}\right]$ such that $R_{2 k} \subset R_{2 k+1}$ is a ramified minimal extension. Next, consider the two cases identified in [6, Proposition 7.6], corresponding to the choices $A:=R_{2 k}(X), B:=R_{2 k+1}(X)$, and $C:=T_{2 k}^{\prime}$, along with $D:=B C$. (Note that the meanings of the symbols $A, B, C$, and $D$ have changed in this paragraph.) The analysis in the preceding paragraph carries over, mutatis mutandis, to provide rings $R_{2 k+2}, T_{2 k+1}^{\prime}$, and $T_{2 k+2}^{\prime}$ with the desired behavior. This completes the proof of the induction step. Since $R(X) \subset S_{1}(X)$ has FCP, we thus find (and fix) a positive integer $n$ such that $R_{2 n}(X)=S_{1}(X)$.

We will show, by a decreasing induction proof, that for each $k$ with $0 \leq k \leq n$, there exists $T_{2 k} \in[R, S]$ such that $T_{2 k}(X)=T_{2 k}^{\prime}$. Once this has been established, taking $T:=T_{0}$ will complete the proof, for then $T(X)=T_{0}(X)=T_{0}^{\prime}=T^{\prime}$, as desired.

We turn to the basis for the decreasing induction, that is, the case $k=n$. Since $R_{2 n}(X) \subset T_{2 n}^{\prime}$ is decomposed, we get that $T_{2 n}^{\prime} \in\left[S_{1}(X), S_{2}(X)\right]$. As the FIP extension $S_{1} \subseteq$ $S_{2}$ is seminormal and infra-integral, we can now apply [4, Proposition 4.17], thus finding some $T_{2 n} \in\left[S_{1}, S_{2}\right]$ such that $T_{2 n}(X)=T_{2 n}^{\prime}$. This completes the proof for the case $k=n$.

Next, for the induction step of the decreasing induction, assume that we have a positive integer $k \leq n$, along with some $T_{2 k} \in[R, S]$ such that $T_{2 k}(X)=T_{2 k}^{\prime}$. Suppose first that $T_{2 k-1}^{\prime}=T_{2 k}^{\prime}$ and $R_{2 k-1}(X)=R_{2 k}(X)$. Then $T_{2 k-1}:=T_{2 k}$ satisfies $T_{2 k-1}(X)=T_{2 k-1}^{\prime}$. We proceed to accomplish the same in the remaining case.

In that remaining case, $T_{2 k-1}^{\prime} \subset T_{2 k}^{\prime}$ and $R_{2 k-1}(X) \subset$ $R_{2 k}(X)$. Then $T_{2 k-1}^{\prime} \subset T_{2 k}^{\prime}$ is a ramified minimal extension, while $R_{2 k}(X) \subset T_{2 k}^{\prime}$ is decomposed minimal. Moreover, $\left(T_{2 k-1}^{\prime}: T_{2 k}^{\prime}\right)$ and $\left(R_{2 k}(X): T_{2 k}^{\prime}\right)$ are incomparable, because $\left(T_{2 k-1}^{\prime}: T_{2 k}^{\prime}\right)$ is a primary nonmaximal ideal of $T_{2 k}^{\prime}$ whose radical is a maximal ideal while $\left(R_{2 k}(X): T_{2 k}^{\prime}\right)$ is a nonmaximal ideal of $T_{2 k}^{\prime}$ that is the intersection of two maximal ideals of $T_{2 k}^{\prime}$. Therefore, by [6, Proposition 6.6(a)], $S^{\prime}:=T_{2 k-1}^{\prime} \cap R_{2 k}(X)$ is such that $S^{\prime} \subset R_{2 k}(X)$ is a ramified minimal extension. This implies that $S^{\prime}=R_{2 k-1}(X)$, since [4, Proposition 4.13] ensures that $\left[R(X), S_{1}(X)\right]$ is linearly ordered by inclusion. Applying Proposition 18 to the chain $R_{2 k-1} \subset R_{2 k} \subset T_{2 k}$ produces $T_{2 k-1} \in\left[R_{2 k-1}, T_{2 k}\right] \subseteq[R, S]$ such that $T_{2 k-1}(X)=T_{2 k-1}^{\prime}$.
The preceding two paragraphs show that, in all (i.e., both) cases, there exists $T_{2 k-1} \in\left[R_{2 k-1}, T_{2 k}\right] \subseteq[R, S]$ such that $T_{2 k-1}(X)=T_{2 k-1}^{\prime}$. We wish to find $T_{2 k-2} \in[R, S]$ such that $T_{2 k-2}(X)=T_{2 k-2}^{\prime}$. If $T_{2 k-2}^{\prime}=T_{2 k-1}^{\prime}$, we may reason as two paragraphs ago, namely, by now taking $T_{2 k-2}:=T_{2 k-1}$. On the other hand, if $T_{2 k-2}^{\prime} \neq T_{2 k-1}^{\prime}$, we may reason as in the preceding paragraph by applying Proposition 18 to produce $T_{2 k-2} \in\left[R_{2 k-2}, T_{2 k-1}\right] \subseteq[R, S]$ such that $T_{2 k-2}(X)=T_{2 k-2}^{\prime}$. This completes the proof of the induction step.

Our next results with a conclusion of the form “ $T$ " $=T(X)$ for some $T$ " will begin with Lemma 25. Several preparatory results are needed first. As usual, if $A$ is a ring, then $A^{2}$ will be used to denote the $\operatorname{ring} A \times A$.

Lemma 20. Let $R \subset S$ be an integral FIP extension with conductor $C$. Let $M \in V_{R}(C)$ and assume that there exist distinct $M_{1}, M_{2} \in V_{S}(C)$ which each lie over $M$. Then $S / M_{1}$ and $S / M_{2}$ are isomorphic as $(R / M)$-algebras if and only if there exists $T \in[R, S]$ such that $T \subset S$ is a decomposed minimal extension with conductor $M_{1} \cap M_{2}$.

Proof. Since $R \subset S$ is an integral extension with FCP, it follows as above from [1, Theorem 4.2(a)] that $R / C$ and $S / C$ are Artinian rings so that $V_{R}(C) \subseteq \operatorname{Max}(R)$ and $V_{S}(C) \subseteq \operatorname{Max}(S)$. Assume first that $S / M_{1}$ and $S / M_{2}$ are isomorphic $(R / M)$ algebras. Identify $S / M_{1}$ with $S / M_{2}$, and let $K$ denote this field. With $N:=M_{1} \cap M_{2}$, we see via the Chinese Remainder Theorem that $S / N \cong K^{2}$. Identifying $S / N$ with $K^{2}$ in this way and viewing $K$ as a subring of $K^{2}$ via the diagonal map (given by $x \mapsto(x, x))$ then allows us to view $K$ as a subring of $S / N$. Consider the canonical surjection $f: S \rightarrow S / N$, and set $T:=f^{-1}(K)$. Note that $T$ is a subring of $S$ which contains $R$ and $N$ and that $T / N \cong S / M_{1}$. It follows that $N \in \operatorname{Max}(T)$ and $N=(T: S)$. Hence, by the criterion in Theorem 4(b), $T \subset S$ is a decomposed minimal extension with conductor $N$.

Conversely, suppose that there exists $T \in[R, S]$ such that $T \subset S$ is a decomposed minimal extension with conductor $M_{1} \cap M_{2}$. Then $M_{1}$ and $M_{2}$ are the only prime (in fact, maximal) ideals of $S$ that lie over $M_{1} \cap M_{2}$ in $T$. By the characterization of "decomposed extensions" in Theorem 4(b), the canonical map $T /\left(M_{1} \cap M_{2}\right) \rightarrow S / M_{i}$ is an isomorphism for $i=1,2$. In particular, $S / M_{1} \cong S / M_{2}$, as desired.

Lemma 21. Let $R \subset S$ be an integral FIP extension. Let $R \subset T^{\prime}$ be a $t$-closed subextension of $R \subset S$ and $R \subset T^{\prime \prime}$ a decomposed minimal subextension of $R \subset S$. Set $T:=T^{\prime} T^{\prime \prime}$. Then, $T^{\prime} \subset T$ is a decomposed minimal extension and $T^{\prime \prime} \subset T$ is an integral t-closed extension.

Proof. Since $R \subset S$ is an integral FIP extension, it follows from Proposition 7(a) that $R \subset T^{\prime}$ can be obtained via a finite (maximal) chain of inert minimal extensions. Also, note that $T^{\prime \prime} \neq T$ since $T^{\prime} \nsubseteq T^{\prime \prime}$ (cf. [1, Lemma 5.6]); similarly, $T^{\prime} \neq T$. Assume first that $(R, M)$ is quasi-local. Pick a maximal (finite) increasing chain $\left\{T_{i}^{\prime}\right\}$ consisting of $n(\geq 1)$ inert extensions going from $R$ to $T^{\prime}$. Set $T_{0}^{\prime}:=R$ and $T_{n}^{\prime}:=T^{\prime}$. We will prove by induction on $n$ that $T^{\prime} \subset T$ is a decomposed 
minimal extension with $\left(T^{\prime}: T\right)=M$ and that $T^{\prime \prime} \subset T$ can be obtained via a chain of $2 n$ inert extensions.

We begin with the induction basis. Then, $n=1$, and so $R \subset T^{\prime}$ is a $t$-closed minimal extension and hence inert (cf. [1, Lemma 5.6]). Thus, it follows from [6, Proposition 7.1(a)] that $T^{\prime} \subset T$ is a decomposed minimal extension with $\left(T^{\prime}: T\right)=$ $M$; and it follows from [6, Proposition 7.1(b)] that $T^{\prime \prime} \subset T$ can be obtained (in two ways) via a chain of 2 inert extensions.

For the induction step, the induction hypothesis states that, for some $k$, with $0<k-1<n, T_{k-1}:=T_{k-1}^{\prime} T^{\prime \prime}$ is such that $T_{k-1}^{\prime} \subset T_{k-1}$ is a minimal decomposed extension with $\left(T_{k-1}^{\prime}: T_{k-1}\right)=M$ and $T^{\prime \prime} \subset T_{k-1}$ can be obtained via a chain of $2 k-2$ inert extensions. Note that $\left(T_{k-1}^{\prime}, M\right)$ is a quasi-local ring, with $T_{k-1}^{\prime} \subset T_{k}^{\prime}$ being inert. Consider $T_{k}:=T_{k}^{\prime} T_{k-1}\left(=T_{k}^{\prime} T_{k-1}^{\prime} T^{\prime \prime}=T_{k}^{\prime} T^{\prime \prime}\right)$. By another application of [6, Proposition 7.1(a)], $T_{k}^{\prime} \subset T_{k}$ is a decomposed minimal extension with $\left(T_{k}^{\prime}: T_{k}\right)=M$; and, by [6, Proposition 7.1(b)], $T_{k-1} \subset T_{k}$ can be obtained (in two ways) via a chain of 2 inert extensions. Thus, $T^{\prime \prime} \subset T_{k}$ can be obtained via a chain of $2 k$ inert extensions (while $R \subset T_{k}^{\prime}$ is obtained via a chain of $k$ inert extensions). This completes the proof of the induction step and establishes the result in case $R$ is quasi-local.

Finally, suppose that $R$ is not quasi-local. Set $M:=(R$ : $\left.T^{\prime \prime}\right)$. Pick $N \in \operatorname{Max}(R)$ with $N \neq M$. Then, $R_{N}=T_{N}^{\prime \prime}$ since $N \neq \mathscr{C}\left(R, T^{\prime \prime}\right)$. It follows that $T_{N}=T_{N}^{\prime} T_{N}^{\prime \prime}=T_{N}^{\prime} R_{N}=T_{N}^{\prime}$. Also, by [6, Proposition 4.6], $R_{M} \subset T_{M}^{\prime \prime}$ is a decomposed minimal extension. We wish to use the above case of a quasilocal base ring to conclude that $T_{M}^{\prime} \subset T_{M}$ is a decomposed minimal extension and $T_{M}^{\prime \prime} \subseteq T_{M}$ is an integral $t$-closed extension. To do so, one must also address the possibility that $R_{M}=T_{M}^{\prime}$; but, in this degenerate case, $T_{M}=T_{M}^{\prime \prime}$, and the assertions follow. Next, note that $R_{N}=T_{N}^{\prime \prime} \subseteq T_{N}=$ $T_{N}^{\prime}$ inherits the $t$-closed property from $R \subset T^{\prime}$. Hence, by [10, Théorème 3.15], the integral extension $T^{\prime \prime} \subset T$ is $t$ closed. Lastly, the assembled information combines with [6, Proposition 4.6] to show that $T^{\prime} \subset T$ is decomposed.

As usual, it will be convenient to call a field extension $K \subseteq$ $L$ simple if $L=K(u)$ for some element $u \in L$.

Lemma 22. Let $K \subset R$ and $K \subset T$ be ring extensions of $a$ field $K$, such that the composite $S:=R T$ exists and $K \subset S$ has FIP. Assume, in addition, that $K \subset R$ is a decomposed minimal extension and $K \subset T$ is an integral $t$-closed extension. Then,

(1) $T$ is a simple field extension of $K$. One can identify $S=$ $T^{2}$ as $T$-algebras and $R=K^{2}$ as $K$-algebras;

(2) let $T^{\prime} \in[K, S]$ be such that $K \subset T^{\prime}$ is $t$-closed with $S=R T^{\prime}$. Then there exist $x \in T$ and $\sigma \in \mathrm{Gal}(T / K)$ such that $T=K[x]$ and $T^{\prime}=K\left[\alpha_{\sigma}\right]$, where $\alpha_{\sigma}:=$ $(x, \sigma(x)) \in T^{2}$. Thus, the diagonal map $T \rightarrow T^{2}$, given by $t \mapsto(t, t)$, allows $T=K[x]$ to be identified with $K[(x, x)]$ as a $K$-algebra.

Proof. (1) By Proposition 8, $T$ is a field. Hence, since $K \subset T$ inherits FIP from $K \subset S$, the Primitive Element Theorem applies to show that the field extension $K \subset T$ is simple. Next, by applying Lemma 21 , we get that $T \subset S$ is a decomposed minimal extension (and that $R \subset S$ is an integral $t$-closed extension). By the classification of the minimal extensions of a field [3, Lemme 1.2], $S \cong T^{2}$ as $T$-algebras and, similarly, $R \cong K^{2}$ as $K$-algebras. There is no harm in identifying $R=K^{2}$ and $S=T^{2}$; nor is there any harm in viewing $K$ (resp., $T$ ) as a subring of $K^{2}$ (resp., of $T^{2}$ ) via the diagonal map.

(2) The case $T^{\prime}=T$ follows from the first assertion in (1) (with $\sigma:=1$ ). Thus, without loss of generality, $T^{\prime} \neq$ $T$. By reasoning as above, Proposition 8 , Lemma 21, and the Primitive Element Theorem can be used to show that $T^{\prime}$ is a field, $T^{\prime} \subset S$ is a decomposed minimal extension, and $T^{\prime}=K[\alpha]$ for some element $\alpha \in T^{\prime}$. Write $\alpha=\left(x_{1}, x_{2}\right)$, for some $x_{1}, x_{2} \in T$. Let $P(X) \in K[X]$ denote the (monic) minimal polynomial of $\alpha$ over $K$. As $P(\alpha)=0$ gives $P\left(x_{1}\right)=$ $0=P\left(x_{2}\right)$, we see that $x_{1}$ and $x_{2}$ each have $P(X)$ as minimal polynomial over $K$. Hence, there exists $\sigma \in \mathrm{Gal}(T / K)$ such that $x_{2}=\sigma\left(x_{1}\right)$, and so $\alpha=\left(x_{1}, \sigma\left(x_{1}\right)\right)$. As

$$
\operatorname{dim}_{K}(S)=\operatorname{dim}_{T}(S) \cdot[T: K]=\operatorname{dim}_{T^{\prime}}(S) \cdot\left[T^{\prime}: K\right]<\infty
$$

and $\operatorname{dim}_{T}(S)=2=\operatorname{dim}_{T^{\prime}}(S)$, we get that $[T: K]=\left[T^{\prime}: K\right]=$ $\operatorname{deg}(P)=\left[K\left[x_{1}\right]: K\right]$. Therefore $T=K\left[x_{1}\right]$, and so the first assertion in (2) holds for $x:=x_{1}$ (and the above $\sigma$ ). The final assertion in (2) is clear.

The following example illustrates the situation in Lemma 22(2) where $T^{\prime} \neq T$.

Example 23. Set $K:=\mathbb{Q}, R:=K^{2}$ and $T:=\mathbb{Q}[i]$. Then $K \subset R$ is a decomposed minimal extension and $K \subset T$ is an integral $t$-closed extension (since $T$ is the field of Gaussian numbers). By calculating vector-space dimensions over $\mathbb{Q}$ as in the proof of Lemma 22, we get that $S:=R T=T^{2}$. Consider $T^{\prime}:=K[(i,-i)] \subseteq T^{2}$. It is clear that $T^{\prime} \neq T$. Moreover, the integral extension $K \subset T^{\prime}$ is $t$-closed; that is, $T^{\prime}$ is a field. To see this, it suffices (in view of integrality) to check that $T^{\prime}$ is a domain. That, in turn, can be shown by straightforward calculations using the fact that $K$ contains no root of $X^{2}+1$. The above data plainly fit the notation of Lemma 22(2), by taking $\sigma$ to be the restriction of complex conjugation and $x:=i$.

The next lemma can be obtained as a consequence of [16, Theorem B(1)] concerning the Samuel cancellation problem that was raised in [17]. We will give a short direct proof of this result for the simple case that we need.

Lemma 24. Let $K \subset L_{1}$ and $K \subset L_{2}$ be two simple algebraic field extensions such that $L_{1}(X)$ and $L_{2}(X)$ are isomorphic as $K$-algebras (for instance, isomorphic as $K(X)$-algebras). Then $L_{1}$ and $L_{2}$ are isomorphic as $K$-algebras.

Proof. Since $K \subset L_{1}$ is a simple extension, there exists $\alpha \in L_{1}$ such that $L_{1}=K(\alpha)$. Let $\psi: L_{1}(X) \rightarrow L_{2}(X)$ be a $K(X)$ isomorphism, and set $\beta:=\psi(\alpha) \in L_{2}(X)$. If $P(Y) \in K[Y]$ is the minimal polynomial of $\alpha$ over $K$, then $P(\beta)=\psi(P(\alpha))=$ 0 . Thus, $\beta$ is algebraic over $K$ and hence also over $L_{2}$. As $L_{2}$ is algebraically closed in $L_{2}(X), \beta \in L_{2}$. If $\beta \in K$, then $\alpha=$ $\psi^{-1}(\beta) \in K$, a contradiction. Thus $\beta \notin K$. Next, note that 
the restriction of $\psi$ to $L_{1}$ gives a $K$-algebra isomorphism from $L_{1}$ onto $K(\beta)$. Thus, it suffices to prove that $K(\beta)=L_{2}$.

The given isomorphism leads to $\left[L_{1}(X): K(X)\right]=$ $\left[L_{2}(X): K(X)\right]<\infty$. By the proof of Proposition 11, $\left[L_{i}(X)\right.$ : $K(X)]=\left[L_{i}: K\right]$ for $i=1,2$. Thus, $\left[L_{2}: K\right]=\left[L_{1}:\right.$ $K]=\operatorname{deg}(P)=[K(\beta): K]$, where the final equality holds because $P(Y)$ is the minimal polynomial of $\beta$ over $K$. As $[K(\beta): K]=\left[L_{2}: K\right]<\infty$ with $K(\beta) \subseteq L_{2}$, we get $K(\beta)=L_{2}$.

Lemma 25. Let $K$ be a field, $K \subset R$ a decomposed minimal extension, and $R \subset S$ an integral t-closed extension such that $K(X) \subset S(X)$ has FIP. Let $T^{\prime} \in[K(X), S(X)]$ be such that $K(X) \subset T^{\prime}$ is t-closed and $S(X)=R(X) T^{\prime}$. Then there exists $T \in[K, S]$ such that $T^{\prime}=T(X)$.

Proof. Since $K(X) \subseteq T^{\prime}$ is an integral $t$-closed extension, $T^{\prime}$ is a field by Lemma 22 (1). Also, as $K(X) \subset R(X)$ is decomposed, Lemma 21 gives that $T^{\prime} \subset S(X)$ is a decomposed minimal extension. Hence, by Theorems 3 and $4,\left(T^{\prime}: S(X)\right)=0=M_{1}^{\prime} \cap M_{2}^{\prime}$, where $M_{1}^{\prime}, M_{2}^{\prime}$ are the two distinct maximal ideals of $S(X)$. It follows that $S$ has exactly two maximal ideals, say, $M_{1}$ and $M_{2}$, and they can be labeled so that $M_{i}^{\prime}=M_{i} S(X)$ for $i=1,2$. Furthermore, $M_{1} \cap M_{2}=0$ since $M_{1}^{\prime} \cap M_{2}^{\prime}=\mathscr{C}\left(T^{\prime}, S(X)\right)=0$. Thus, $S \cong S / M_{1} \times$ $S / M_{2}$ by the Chinese Remainder Theorem. The following isomorphisms of $T^{\prime}$-algebras, and hence of $K(X)$-algebras, hold by Theorem 4: $S(X) / M_{1} S(X) \cong T^{\prime} \cong S(X) / M_{2} S(X)$. Thus, $\left(S / M_{1}\right)(X) \cong T^{\prime} \cong\left(S / M_{2}\right)(X)$.

Set $N_{i}:=M_{i} \cap R$ for $i=1,2$. One can see that $N_{1} \neq N_{2}$ via Theorem 3(a) since there is a finite chain of inert extensions going from $R$ to $S$. Also, $N_{1} \cap N_{2}=0$; and since $K \subset R$ is a decomposed minimal extension, $N_{1}$ and $N_{2}$ are the two maximal ideals of $R$. As $R / N_{i} \cong K$ for each $i$, the Chinese Remainder Theorem gives $R \cong K^{2}$.

We wish to use Lemma 22(1) to show that $K \cong R / N_{i} \rightarrow$ $S / M_{i}$ is a simple field extension for each $i$. First, note that since $R \subset S$ is integral, with $M_{i}$ the only maximal ideal of $S$ lying over $N_{i}$, we can identify $S_{N_{i}}=S_{M_{i}}$. Thus, if $R_{N_{i}}=S_{N_{i}}$ canonically, we see by passing to residue fields that the field extension $R / N_{i} \rightarrow S / M_{i}$ is an equality and hence a simple field extension. Therefore, we may suppose that $R_{N_{i}} \subset S_{N_{i}}$. As $R / N_{i} \rightarrow S / M_{i}$ is an FIP (hence integral) extension, it will be enough to show that it is $t$-closed. By [10, Theorem 3.15], the $t$-closed hypothesis implies that $R_{N_{i}} \subset S_{N_{i}}$ is $t$-closed with conductor $N_{i} R_{N_{i}}$. Thus, $R_{N_{i}} / N_{i} R_{N_{i}} \subseteq S_{N_{i}} / N_{i} R_{N_{i}}$ is $t$-closed. Also, since $R_{N_{i}} \subseteq S_{N_{i}}$ can be obtained via a chain of inert extensions, $N_{i} R_{N_{i}}$ must be a maximal ideal of $S_{N_{i}}$ (necessarily, $N_{i} S_{N_{i}}=M_{i} S_{N_{i}}$ ). Of course, $R_{N_{i}} / N_{i} R_{N_{i}}=R / N_{i}$ canonically. Thus, it suffices to show that $S_{N_{i}} / N_{i} R_{N_{i}}=S / M_{i}$ canonically. As we have identified $S_{N_{i}}=S_{M_{i}}$, equating maximal ideals gives that $\left(N_{i} R_{N_{i}}=\right) M_{i} S_{N_{i}}=M_{i} S_{M_{i}}$, and so $S_{N_{i}} / N_{i} R_{N_{i}}=$ $S_{M_{i}} / M_{i} S_{M_{i}}=S / M_{i}$, as desired.

We have seen that $S / M_{1}$ and $S / M_{2}$ are each simple field extensions of $K$ and that $\left(S / M_{1}\right)(X) \cong\left(S / M_{2}\right)(X)$ as algebras over $K(X)$. Hence, by Lemma $24, S / M_{1}$ and $S / M_{2}$ are isomorphic as $K$-algebras. It follows, by applying Lemma 20 to $K \subset S$, that there exists $U \in[K, S]$ such that $U \subset S$ is a decomposed minimal extension with $0=M_{1} \cap M_{2}=(U$ : $S)=\mathscr{C}(U, S) \in \operatorname{Max}(U)$. Therefore, $U$ is a field. So, by [3, Lemme 1.2], we can identify $S$ with $U^{2}$.

Observe that $U \cap R=K$ by the minimality of $K \subset R$, $K \subset U$ is $t$-closed by Proposition 8 , and $U R=S$ by the minimality of $U \subset S$. Also, $K(X) \subseteq U(X)$ is $t$-closed and $U(X) R(X)=S(X)$. Moreover, $K \subset U$ has FIP. In fact, by applying Lemma 22(2), we get an element $a \in U$ such that $U=K[a]$ (which can be identified with $K[(a, a)]$ ) and $\sigma \in \operatorname{Gal}(U(X) / K(X))$ such that $T^{\prime}=K(X)\left[a_{\sigma}\right]$, where $a_{\sigma}:=(a, \sigma(a)) \in S(X)$. (Note also that $U(X)=$ $K[a](X)=K(X)[a]$.) As the isomorphism $\operatorname{Gal}(U / K) \cong$ $\operatorname{Gal}(U(X) / K(X))$ from Lemma 13 gives that $a_{\sigma} \in S$, it follows that $T^{\prime}=K\left[a_{\sigma}\right](X)$. In particular, $T:=K\left[a_{\sigma}\right] \in[K, S]$ satisfies $T(X)=T^{\prime}$.

Proposition 26. Let $(R, M)$ be a quasi-local ring and let $R \subset S$ be an integral extension such that $R(X) \subset S(X)$ is an FIP extension. Assume that $R \subset{ }_{S}^{t} R$ is a decomposed minimal extension. Let $T^{\prime} \in[R(X), S(X)]$ be such that $R(X) \subset T^{\prime}$ is a $t$-closed extension. Then there exists $T \in[R, S]$ such that $T^{\prime}=T(X)$.

Proof. Set $S_{2}:={ }_{S}^{t} R$ (whence $S_{2}(X)={ }_{S(X)}^{t} R(X)$ ) and $U^{\prime}:=$ $S_{2}(X) T^{\prime}$. Then, by Lemma 21, $T^{\prime} \subset U^{\prime}$ is a decomposed minimal extension and $S_{2}(X) \subseteq U^{\prime}$ is an integral $t$ closed extension. It follows that $U^{\prime} \in\left[S_{2}(X), S(X)\right]$, and so Theorem 12 supplies $U \in\left[S_{2}, S\right]$ such that $U^{\prime}=U(X)$. As $M$ is a common ideal of $R$ and $S_{2}$, we have that $M R(X)$ is a common ideal of $R(X), S_{2}(X)$, and $T^{\prime}$. We claim that $M$ is also an ideal of $U$ (and so $M R(X)$ is an ideal of $U(X)$ ).

To prove the claim, it suffices to show that $M$ is an ideal of $S$ (since $U \in[R, S]$ ). Without loss of generality, we may assume $\left(S_{2}: S\right) \neq M$. Now, since $R \subset S_{2}$ is a decomposed minimal extension, we can write $M=M_{1} \cap M_{2}$, where $\operatorname{Max}\left(S_{2}\right)=\left\{M_{1}, M_{2}\right\}$. Hence, by Proposition 7(2), $\left(S_{2}: S\right)$ is one of $M, M_{1}, M_{2}$. By the above comment, we may suppose that $\left(S_{2}: S\right)=M_{1}$. Then, since $S_{2} \subset S$ is integral $t$-closed, we get that $M_{1}$ is a maximal ideal of $S$. (Note that $S_{2} \neq S$ because the hypothesis on $T^{\prime}$ ensures that $R(X) \subset S(X)$ is not a decomposed minimal extension.) Let $N_{2}$ denote the maximal ideal of $S$ lying over $M_{2}$. Then $M=M_{1} \cap M_{2}=$ $M_{1} \cap S_{2} \cap N_{2}=M_{1} \cap N_{2}$, which is an ideal of $S$, thus completing the proof of the above claim.

Set $K:=R / M, R^{\prime}:=S_{2} / M$, and $S^{\prime}:=U / M$. It follows that $T^{\prime} / M R(X) \in\left[K(X), S^{\prime}(X)\right]$, with $K(X) \subseteq T^{\prime} / M R(X)$ $t$-closed and $S^{\prime}(X)=R^{\prime}(X)\left(T^{\prime} / M R(X)\right)$. Then, since $K \subset$ $R^{\prime}$ is decomposed, Lemma 25 supplies $T^{\prime \prime} \in\left[K, S^{\prime}\right]$ such that $T^{\prime \prime}(X)=T^{\prime} / M R(X)$. Of course, $T^{\prime \prime}=T / M$ for some $T \in[R, U]$. Then $T(X) / M R(X)=(T / M)(X)=T^{\prime \prime}(X)=$ $T^{\prime} / M R(X)$ canonically, and so $T(X)=T^{\prime}$.

The next lemma will be used to show the applicability of Proposition 26 to the proof of Proposition 28.

Lemma 27. Let $(R, M)$ be a quasi-local ring, let $R \subset R_{1}$ be a decomposed extension, and let $R \subset T$ be an integral FIP $t$ closed extension such that $S:=R_{1} T$ exists. Then $R_{1}$ is the $t$ closure of $R$ in $S$. 
Proof. By the hypothesis on $R \subset T$, there exists a finite increasing chain $\left\{T_{i}\right\} \subseteq[R, T]$ arising from inert minimal extensions $T_{i} \subset T_{i+1}$ such that $T_{1}=R$ and $T_{n}=T$. Since each of these extensions is inert, each $T_{i}$ is a quasi-local ring with maximal ideal $M$. Using [6, Proposition 7.1(a), (b)], we can inductively construct an increasing chain $\left\{R_{i}\right\} \subseteq\left[R_{1}, S\right]$ such that for each $i \geq 1, T_{i} \subset R_{i}$ is a decomposed minimal extension and $R_{1} \subset R_{i}$ is $t$-closed, with $R_{i}=R_{i-1} T_{i}=R_{1} T_{i}$. (Note that $R_{1} \subset R_{i}$ is $t$-closed since each step $R_{i} \subset R_{i+1}$ results via a chain $R_{i} \subset A_{i} \subset R_{i+1}$, where both $R_{i} \subset A_{i}$ and $A_{i} \subset R_{i+1}$ are inert minimal extensions.) Consequently, $R_{1}$ is the $t$-closure of $R$ in $R_{i}$. Taking $i=n$, we see that $R_{n}=R_{1} T_{n}=R_{1} T=S$. Thus, $R_{1}$ is the $t$-closure of $R$ in $S$.

Proposition 28. Let $R \subset S$ be an integral ring extension such that $R(X) \subset S(X)$ has FIP. Let $T^{\prime} \in[R(X), S(X)]$ and $R_{1} \in$ $[R, S]$ be such that $R \subset R_{1}$ is a decomposed minimal extension, $R(X) \subset T^{\prime}$ is a $t$-closed extension, and $S(X)=T^{\prime} R_{1}(X)$. Then there exists $T \in[R, S]$ such that $T^{\prime}=T(X)$.

Proof. Let $M:=\left(R: R_{1}\right)$. Then $R_{M} \subset\left(R_{1}\right)_{M}$ is a decomposed minimal extension. We wish to show that there exists $T^{\prime \prime} \in\left[R_{M}, S_{M}\right]$ such that $T_{M R(X)}^{\prime}=T^{\prime \prime}(X)$. Without loss of generality, $R(X)_{M R(X)} \subset T_{M R(X)}^{\prime}$. Note that this extension is $t$-closed. In addition, $S(X)_{M R(X)}=\left(T^{\prime} R_{1}(X)\right)_{M R(X)}$. Thus, the desired $T^{\prime \prime}$ will be supplied by Proposition 26, provided that we first show that the $t$-closure of $R_{M}$ in $S_{M}$ is $\left(R_{1}\right)_{M}$. In fact, by applying the preceding lemma to the extensions $R(X)_{M R(X)} \subset R_{1}(X)_{M R(X)}$ and $R(X)_{M R(X)} \subset T_{M R(X)}^{\prime}$, we get that $R_{1}(X)_{M R(X)}$ is the $t$-closure of $R_{M}(X)$ in $S_{M}(X)$, whence $\left(R_{1}\right)_{M}$ is the $t$-closure of $R_{M}$ in $S_{M}$. Thus, the applicability of Proposition 26 has been validated. With the suitable $T^{\prime \prime}$ in hand, we next use [1, Theorem 3.6], the upshot being (a unique) $T \in[R, S]$ such that $T_{M}=T^{\prime \prime}$ and $T_{N}=S_{N}$ for each $N \in \operatorname{Max}(R)$ such that $N \neq M$. But $R_{N}=\left(R_{1}\right)_{N}$ for any such $N$, since $M=\mathscr{C}\left(R, R_{1}\right)$, whence $T(X)_{N R(X)}=T_{N}(X)=$

$$
S_{N}(X)=T_{N R(X)}^{\prime}\left(\left(R_{1}\right)_{N}(X)\right)=T_{N R(X)}^{\prime} R_{N}(X)=T_{N R(X)}^{\prime} .
$$

As $T(X)_{M R(X)}=T_{M}(X)=T^{\prime \prime}(X)=T_{M R(X)}^{\prime}$, globalization yields that $T(X)=T^{\prime}$.

Proposition 29. Let $(R, M)$ be a quasi-local ring and $R \subset S$ an integral seminormal FIP extension such that $R \subset{ }_{S}^{t} R$. Let $R(X) \subset T^{\prime}$ be an inert minimal subextension of $R(X) \subset S(X)$. Then there exists $T \in[R, S]$ such that $T^{\prime}=T(X)$.

Proof. By hypothesis, ${ }_{S}^{+} R=R$. Set $S_{2}:={ }_{S}^{t} R$. Then $R(X)=$ ${ }_{S(X)}^{+} R(X)$ and $S_{2}(X)={ }_{S(X)}^{t} R(X)$ by [4, Lemma 3.15]. Since $R \subset S$ has FIP, there exists a finite chain of rings $R=R_{0} \subseteq$ $\cdots R_{i} \subset R_{i+1} \subseteq \cdots \subseteq R_{n}=S_{2}$ such that $R_{i} \subset R_{i+1}$ is a decomposed minimal extension for each $i \in\{0, \ldots, n-1\}$. Note that $n \geq 1$, since $R \subset S_{2}$. By induction on $i$ where $0 \leq i \leq n$, we are going to construct a maximal finite chain of rings $\left\{T_{i}^{\prime}\right\} \subseteq\left[T^{\prime}, S(X)\right]$ such that $R_{i}(X) \subset T_{i}^{\prime}$ is a $t$ closed extension for each $i$. The induction statement for $i$ is formulated as follows: there exists $T_{i}^{\prime} \in\left[T^{\prime}, S(X)\right]$ such that
$R_{i}(X) \subset T_{i}^{\prime}$ is a $t$-closed extension. The induction basis (the case for $i=0$ ) can be established by taking $T_{0}^{\prime}:=T^{\prime}$, for then $R_{0}(X) \subset T^{\prime}$ (being inert) is $t$-closed.

For the induction step, assume the induction statement for some integer $i$ where $0 \leq i<n$. By hypothesis, there exists $T_{i}^{\prime} \in\left[T^{\prime}, S(X)\right]$ such that $R_{i}(X) \subset T_{i}^{\prime}$ is $t$-closed. Since $R_{i} \subset R_{i+1}$ is a decomposed minimal extension, so is $R_{i}(X) \subset$ $R_{i+1}(X)$, by [4, Theorem 3.4]. Set $T_{i+1}^{\prime}:=T_{i}^{\prime} R_{i+1}(X)$. Then, by Lemma $21, T_{i}^{\prime} \subset T_{i+1}^{\prime}$ is a minimal decomposed extension and $R_{i+1}(X) \subset T_{i+1}^{\prime}$ is a(n integral) $t$-closed extension. This completes the proof of the induction step. Thus, we have constructed the desired chain $\left\{T_{i}^{\prime}\right\}$. Note also that $T_{i}^{\prime} \subset T_{i+1}^{\prime}$ is a minimal decomposed extension whenever $0 \leq i \leq n-1$ and that $T_{n}^{\prime} \in\left[S_{2}(X), S(X)\right]$.

We will show, by a decreasing induction proof, that for each $i$ with $0 \leq i \leq n$, there exists $T_{i} \in[R, S]$ such that $T_{i}(X)=T_{i}^{\prime}$. Once this has been established, taking $T:=T_{0}$ will complete the proof, for then $T(X)=T_{0}(X)=T_{0}^{\prime}=T^{\prime}$, as desired.

We turn to the basis for the decreasing induction, that is, the case $i=n$. As $T_{n}^{\prime} \in\left[S_{2}(X), S(X)\right]$ and $S_{2} \subseteq S$ is an integral $t$-closed extension, Theorem 12 provides $T_{n} \in\left[S_{2}, S\right]$ such that $T_{n}(X)=T_{n}^{\prime}$. (In fact, Theorem 12 can be applied if $S_{2} \subset S$, but the assertion is clear if $S_{2}=S$.) This completes the proof for the case $i=n$.

Next, for the induction step of the decreasing induction, assume that we have a non-negative integer $i<n$, along with some $T_{i+1} \in[R, S]$ such that $T_{i+1}(X)=T_{i+1}^{\prime}$. Recall that $T_{i}^{\prime} \in\left[R_{i}(X), T_{i+1}(X)\right]$, with $R_{i} \subset R_{i+1}$ a decomposed minimal extension and $R_{i}(X) \subset T_{i}^{\prime}$ a $t$-closed extension such that $T_{i+1}(X)=T_{i}^{\prime} R_{i+1}(X)$. Therefore, it follows from Proposition 28 that there exists $T_{i} \in\left[R_{i}, T_{i+1}\right] \subseteq[R, S]$ such that $T_{i}^{\prime}=T_{i}(X)$. This completes the proof.

We are now able to give a first answer to the main questions for the case of a quasi-local base ring.

Proposition 30. Let $(R, M)$ be a quasi-local ring and $R \subset S$ a ring extension such that $R(X) \subset S(X)$ has FIP. Let $T^{\prime} \epsilon$ $[R(X), S(X)]$ be such that $R(X) \subset T^{\prime}$ is a minimal extension. Then there exists some $T \in[R, S]$ such that $T(X)=T^{\prime}$.

Proof. Since $R(X) \subset T^{\prime}$ is a minimal extension, its crucial ideal must be $M R(X)$, the only maximal ideal of $R(X)$. Set $S_{1}:={ }_{S}^{+} R$ and $S_{2}:={ }_{S}^{t} R$. Then $S_{1}(X)={ }_{S(X)}^{+} R(X)$ and $S_{2}(X)={ }_{S(X)}^{t} R(X)$, by [4, Lemma 3.15]. We complete the proof by considering each of the four types of minimal extensions.

First, assume that $R(X) \subset T^{\prime}$ is a flat epimorphism. As $R(X)$ is quasi-local, [4, Lemma 4.9] shows that there cannot exist an integral minimal extension of $T^{\prime}$ inside $S(X)$. Hence, by FCP, $T^{\prime}$ must be integrally closed in $S(X)$. Consequently, $R(X)$ is integrally closed in $S(X)$, and so $R$ is integrally closed in $S$. Then [4, Proposition 4.4] supplies $T$ with the asserted properties.

Next, assume that $R(X) \subset T^{\prime}$ is ramified. Then $T^{\prime} \in$ [R(X), $\left.S_{1}(X)\right]$. As $R \subseteq S_{1}$ is subintegral, [4, Proposition 4.14] supplies the desired $T$. 
Next, assume that $R(X) \subset T^{\prime}$ is decomposed. If $R=S_{1}$, then $T^{\prime} \in\left[S_{1}(X), S_{2}(X)\right]$, and so [4, Proposition 4.17] supplies the desired $T$. On the other hand, if $R \subset S_{1}$, then FCP leads to $R_{1} \in\left[R, S_{1}\right]$ such that $R \subset R_{1}$ is a ramified minimal extension; in this subcase, Proposition 19 supplies the desired $T$.

We may suppose henceforth that we are in the final case, namely, where $R(X) \subset T^{\prime}$ is inert. Then $(R(X)$ : $\left.T^{\prime}\right)=M R(X)$. If $S_{2}=R$, then $T^{\prime} \in\left[S_{2}(X), \bar{R}(X)\right]$, and so Theorem 12 supplies the desired $T$. The same conclusion holds if $S_{1}=R$, thanks to Proposition 29. Thus, without loss of generality, $R \subset S_{1}$.

As $R \subset S_{1}$ satisfies FCP, there exists $R_{1} \in\left[R, S_{1}\right]$ such that $R \subset R_{1}$ is a ramified minimal extension. Set $K:=$ $R(X) / M R(X), L:=T^{\prime} / M R(X), R_{1}^{\prime}:=R_{1}(X) / M R(X)$, and $L^{\prime}:=L R_{1}^{\prime}$. Since $R(X) \subset T^{\prime}$ is inert, $K \subset L$ is a minimal field extension; also, $K \subset R_{1}^{\prime}$ inherits the "ramified minimal extension" property from $R(X) \subset R_{1}(X)$. Therefore, by [6, Proposition 7.4], $L \subset L^{\prime}$ is a ramified minimal extension. Hence, $L^{\prime}\left(\cong L[Y] /\left(Y^{2}\right)\right)$ is a quasi-local nonreduced ring which is a ring extension of the infinite field $K$. As $K \subset L^{\prime}$ has FIP, an application of [2, Theorem 3.8] shows that there exists $\alpha \in L^{\prime}$ such that $L^{\prime}=K(\alpha)$ and $\alpha^{3}=0$. Consequently, $\operatorname{dim}_{K}\left(L^{\prime}\right) \leq 3$. Moreover, since $R_{1}^{\prime} \nsubseteq L$, we have that $L \neq L^{\prime}$. Then, since $L$ is a field, $\operatorname{dim}_{L}\left(L^{\prime}\right) \geq 2$ and

$$
\operatorname{dim}_{K}\left(L^{\prime}\right)=[L: K] \cdot \operatorname{dim}_{L}\left(L^{\prime}\right) \geq 2 \cdot 2=4,
$$

a contradiction (to the possibility that $R \subset S_{1}$ ). The case analysis is complete.

We next remove the "quasi-local" hypothesis from the preceding proposition.

Proposition 31. Let $R \subset S$ be a ring extension such that $R(X) \subset S(X)$ has FIP. Let $R(X) \subset T^{\prime}$ be a minimal subextension of $R(X) \subset S(X)$. Then there exists $T \in[R, S]$ such that $R \subset T$ is a minimal extension and $T^{\prime}=T(X)$.

Proof. Since $R(X) \subset S(X)$ is an FIP extension, so is $R \subset S$ and $\operatorname{MSupp}(S(X) / R(X))=\{M R(X) \mid M \in \operatorname{MSupp}(S / R)\}$ is a finite set, by [4, Proposition 3.2 and Lemma 3.3] and [1, Corollary 3.2]. Hence, by the hypothesis on $T^{\prime}$, there exists $M \in \operatorname{MSupp}(S / R)$ such that $\mathscr{C}\left(R(X), T^{\prime}\right)=M R(X)$. Moreover, $R_{M}(X)=R(X)_{M R(X)} \subset T_{M R(X)}^{\prime}$ is a minimal extension, while $R(X)_{M^{\prime} R(X)}=T_{M^{\prime} R(X)}^{\prime}$ for each $M^{\prime} \in$ $\operatorname{Max}(R)$ such that $M^{\prime} \neq M$.

By the case of a quasi-local base ring (Proposition 30), there exists $T^{\prime \prime} \in\left[R_{M}, S_{M}\right]$ such that $T_{M R(X)}^{\prime}=T^{\prime \prime}(X)$. Since $R_{M}(X) \subset T^{\prime \prime}(X)$ is a minimal extension, it follows from Theorem 6 that $R_{M} \subset T^{\prime \prime}$ is a minimal extension.

Let $\pi: S \rightarrow S_{M}$ denote the canonical ring homomorphism, and set $T_{1}:=\pi^{-1}\left(T^{\prime \prime}\right)$. Then $T_{1} \in[R, S]$ and $\left(T_{1}\right)_{M}=T^{\prime \prime}$. Since $R_{M} \subset\left(T_{1}\right)_{M}$ is a minimal extension, $M \in$ $\operatorname{MSupp}\left(T_{1} / R\right)$. Pick a finite chain of rings $R=R_{0} \subset \cdots \subset R_{i} \subset$ $\cdots \subset R_{n}=T_{1}$ such that $R_{i} \subset R_{i+1}$ is a minimal extension for each $i \in\{0, \ldots, n-1\}$. Then, by [1, Corollary 3.2], there exists an index $j$ such that the minimal extension $R_{j} \subset R_{j+1}$ satisfies $M=\mathscr{C}\left(R_{j}, R_{j+1}\right) \cap R$. By localizing the members of the above chain at $M$ and using the minimality of $R_{M} \subset\left(T_{1}\right)_{M}$, we see that $R_{M}=\left(R_{j}\right)_{M}$ and $\left(T_{1}\right)_{M}=\left(R_{j+1}\right)_{M}$. Then $M \notin$ $\operatorname{Supp}\left(R_{j} / R\right)$ and $\operatorname{MSupp}\left(R_{j+1} / R_{j}\right)=\{M\}$. (Indeed, $\left(R_{j}\right)_{M^{\prime}}=$ $\left(R_{j+1}\right)_{M^{\prime}}$ for each $M^{\prime} \in \operatorname{Max}(R)$ such that $M^{\prime} \neq M$.) It follows that $\operatorname{MSupp}\left(R_{j} / R\right)$ and $\operatorname{MSupp}\left(R_{j+1} / R_{j}\right)$ are disjoint. As the support of any module is stable under specialization (i.e., under passage to a larger prime ideal), it follows that $\operatorname{Supp}\left(R_{j} / R\right)$ and $\operatorname{Supp}\left(R_{j+1} / R_{j}\right)$ are disjoint. Therefore, if $R \neq$ $R_{j}$ (i.e., if $j \neq 0$ ), we can apply the statement of $[18$, Lemma 2.9] to obtain a unique $T \in\left[R, R_{j+1}\right]$ such that $T \cap R_{j}=$ $R$ and $T R_{j}=R_{j+1}$. On the other hand, if $R=R_{j}$, then the same properties hold for $T:=R_{j+1}$. Thus, in any case, $\left(T_{1}\right)_{M}=\left(R_{j+1}\right)_{M}=T_{M}\left(R_{j}\right)_{M}=T_{M} R_{M}=T_{M}$; and, for all $M^{\prime} \in \operatorname{Max}(R)$ with $M^{\prime} \neq M$,

$$
R_{M^{\prime}}=T_{M^{\prime}} \cap\left(R_{j}\right)_{M^{\prime}}=T_{M^{\prime}} \cap\left(R_{j+1}\right)_{M^{\prime}}=T_{M^{\prime}} .
$$

According to [1, Theorem 3.6(a)], to show that $T(X)=T^{\prime}$ (and thus complete the proof), it suffices to prove that $T(X)$ and $T^{\prime}$ give the same localizations at each maximal ideal of $R(X)$. We have

$$
T(X)_{M R(X)}=T_{M}(X)=\left(T_{1}\right)_{M}(X)=T^{\prime \prime}(X)=T_{M R(X)}^{\prime} ;
$$

and if $M^{\prime} \in \operatorname{Max}(R)$ with $M^{\prime} \neq M$, then

$$
\begin{aligned}
T(X)_{M^{\prime} R(X)} & =\left(T_{M^{\prime}}\right)(X)=\left(R_{M^{\prime}}\right)(X) \\
& =R(X)_{M^{\prime} R(X)}=T_{M^{\prime} R(X)}^{\prime}
\end{aligned}
$$

We can now obtain the desired result.

Theorem 32. Let $R \subset S$ be a ring extension such that $R(X) \subset$ $S(X)$ has FIP. Then the function $\varphi:[R, S] \rightarrow[R(X), S(X)]$, defined by $T \mapsto T(X)$, is an order-isomorphism. Consequently, $|[R(X), S(X)]|=|[R, S]|$ and $\ell[R, S]=\ell[R(X), S(X)]$.

Proof. As explained at the beginning of this section, it follows from [4, Lemma 3.1(d)] that it suffices to show that $\varphi$ is surjective. For any $T^{\prime} \in[R(X), S(X)]$, there exists a finite maximal chain of rings $R(X)=R_{0}^{\prime} \subset \cdots \subset R_{i}^{\prime} \subset \cdots \subset R_{n}^{\prime}=T^{\prime}$ such that each $R_{i}^{\prime} \subset R_{i+1}^{\prime}$ is minimal, because $R(X) \subset S(X)$ has FCP. We show by induction that for each $i$, there exists $R_{i} \in$ $[R, S]$ such that $R_{i}^{\prime}=R_{i}(X)$. By definition, we have $R_{0}^{\prime}=R(X)$. Assume that, for some specific $i<n$, there exists $R_{i}^{\prime} \in[R, S]$ such that $R_{i}^{\prime}=R_{i}(X)$. Observe that $R_{i}(X) \subset S(X)$ has FIP and $R_{i}(X) \subset R_{i+1}^{\prime}$ is minimal. Therefore, by Proposition 31, there exists $R_{i+1} \in\left[R_{i}, S\right]$ such that $R_{i+1}^{\prime}=R_{i+1}(X)$ (and $R_{i} \subset R_{i+1}$ is minimal). This completes the induction argument. The case $i:=n-1$ shows that there exists $T \in[R, S]$ such that $T^{\prime}=T(X)$; that is, $\varphi$ is surjective.

Remark 33. Theorem 32 completely answers the questions about cardinality and order that were raised in [4, Remark 4.18(a)]. 


\section{Conflict of Interests}

The authors declare that there is no conflict of interests regarding the publication of this paper.

\section{References}

[1] D. E. Dobbs, G. Picavet, and M. Picavet-L'Hermitte, "Characterizing the ring extensions that satisfy FIP or FCP," Journal of Algebra, vol. 371, pp. 391-429, 2012.

[2] D. D. Anderson, D. E. Dobbs, and B. Mullins, "The primitive element theorem for commutative algebras," Houston Journal of Mathematics, vol. 25, pp. 603-623, 1999.

[3] D. Ferrand and J.-P. Olivier, "Homomorphismes minimaux d'anneaux," Journal of Algebra, vol. 16, pp. 461-471, 1970.

[4] D. E. Dobbs, G. Picavet, and M. Picavet-L'Hermitte, “Transfer results for the FIP and FCP properties of ring extensions," Communications in Algebra. To appear.

[5] L. I. Dechéne, Adjacent extensions of rings, University of California at Riverside, Riverside, Calif, USA, 1978.

[6] D. E. Dobbs, G. Picavet, M. Picavet-L'Hermitte, and J. Shapiro, "On intersections and composites of minimal ring extensions," JP Journal of Algebra, Number Theory and Applications, vol. 26, no. 2, pp. 103-158, 2012.

[7] G. Picavet and M. Picavet-L'Hermitte, "About minimal morphisms," in Multiplicative Ideal Theory in Commutative Algebra, pp. 369-386, Springer, New York, NY, USA, 2006.

[8] D. Lazard, "Autour de la platitude," Bulletin de la Société Mathématique de France, vol. 97, pp. 81-128, 1969.

[9] D. E. Dobbs, B. Mullins, G. Picavet, and M. Picavet-L'Hermitte, "On the FIP property for extensions of commutative rings," Communications in Algebra, vol. 33, no. 9, pp. 3091-3119, 2005.

[10] G. Picavet and M. Picavet-L'Hermitte, "Morphismes $t$-clos," Communications in Algebra, vol. 21, no. 1, pp. 179-219, 1993.

[11] N. Bourbaki, Algèbre, chapitres 4-7, Masson, Paris, France, 1981.

[12] C. Greither, Cyclic Galois Extensions of Commutative Rings, vol. 1534 of Lecture Notes in Mathematics, Springer, Berlin, Germany, 1992.

[13] D. D. Anderson, D. F. Anderson, and R. Markanda, "The rings $R(X)$ and $R\langle X\rangle$," Journal of Algebra, vol. 95, no. 1, pp. 96-115, 1985.

[14] O. Zariski and P. Samuel, Commutative Algebra, vol. 1, D. Van Nostrand, Princeton, NJ, USA, 1963.

[15] A. Grothendieck and J. Dieudonné, Éléments de Géométrie Algébrique IV, No. 20, Presses Universitaires de France, Paris, France, 1964.

[16] M. C. Kang, "A note on the birational cancellation problem," Journal of Pure and Applied Algebra, vol. 77, no. 2, pp. 141-154, 1992.

[17] P. Samuel, "Some Remarks on Lüroth's Theorem," Memoirs of the College of Science, University of Kyoto A, vol. 27, no. 3, pp. 223-224, 1953.

[18] G. Picavet and M. Picavet-L'Hermitte, "Prüfer and Morita hulls of FCP extensions," Communications in Algebra. To appear. 


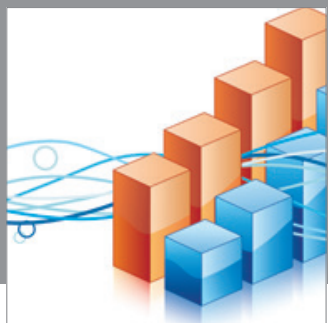

Advances in

Operations Research

mansans

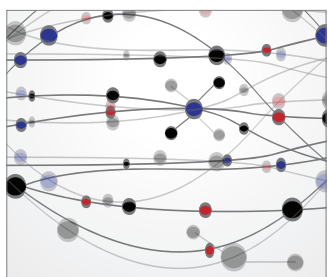

The Scientific World Journal
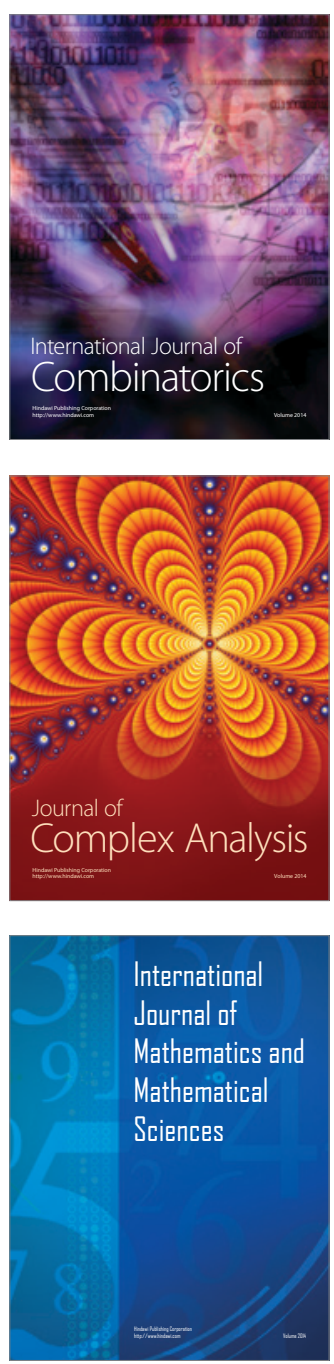
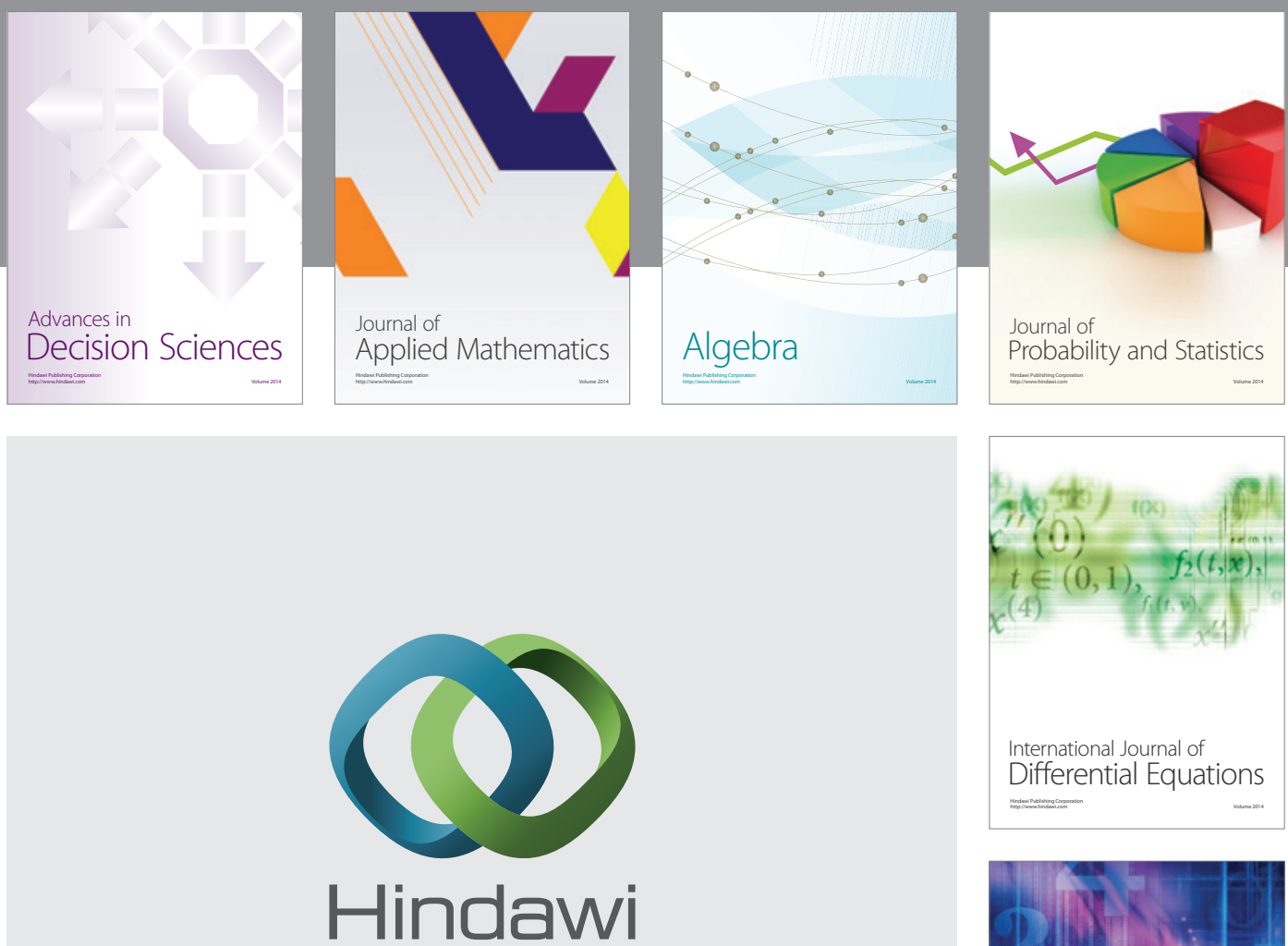

Submit your manuscripts at http://www.hindawi.com
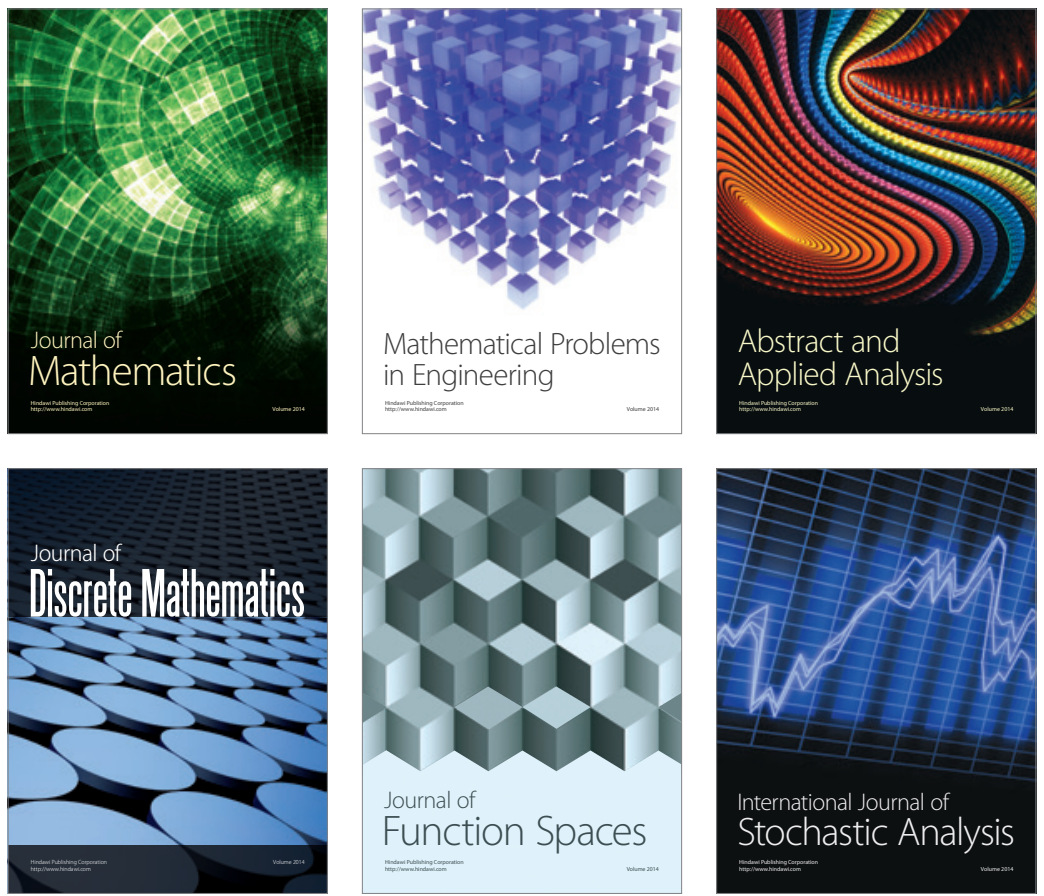

Journal of

Function Spaces

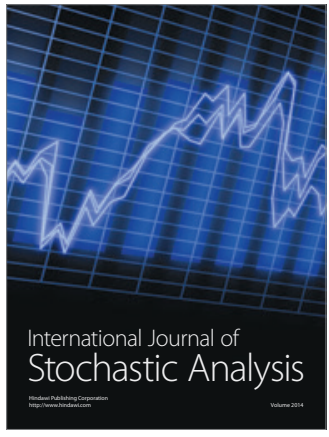

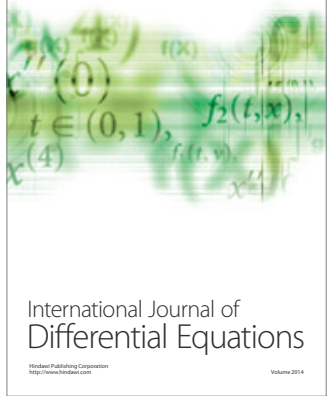
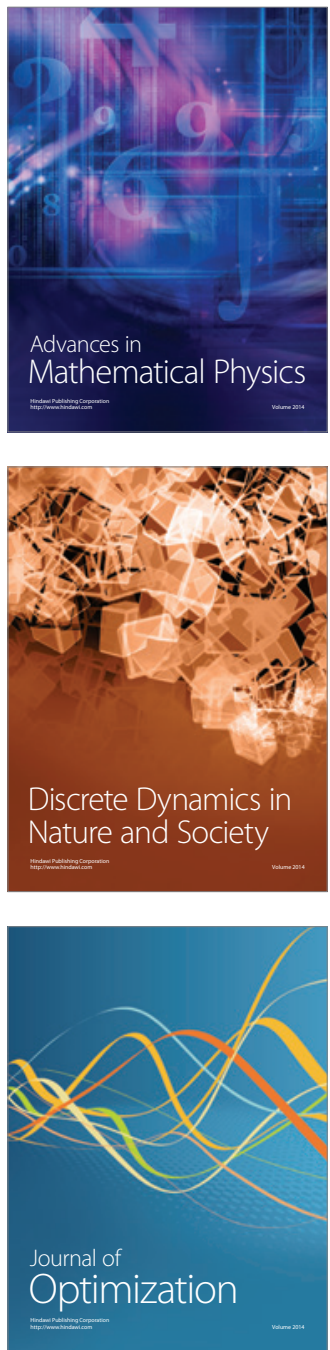